\title{
A weak-scattering model for turbine-tone haystacking outside the cone of silence
}

by

Christopher J. Powles, Brian J. Tester, and Alan McAlpine

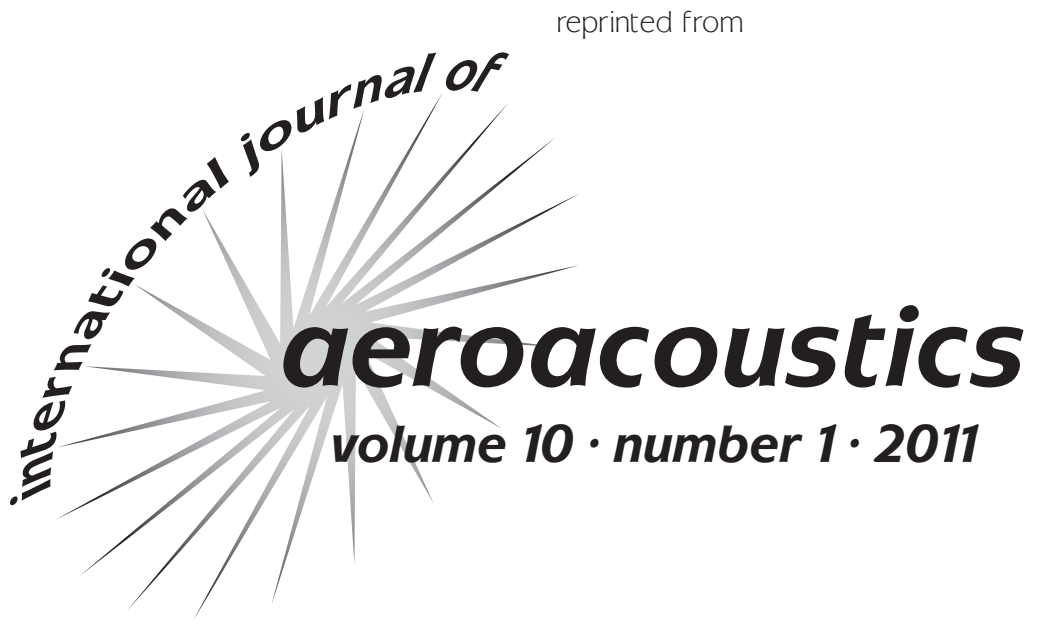

published by MULTI-SCIENCE PUBLISHING CO. LTD.,

5 Wates Way, Brentwood, Essex, CM15 9TB UK

E-MAIL: mscience@globalnet.co.uk

WEBSITE: Www.multi-science.co.uk 


\title{
A weak-scattering model for turbine-tone haystacking outside the cone of silence
}

\author{
By Christopher J. Powles, Brian J. Tester, and Alan McAlpine \\ Institute of Sound and Vibration Research, University of Southampton, Highfield, Southampton, \\ Hampshire, SO17 1BJ, UK \\ E-mail: cp1@isvr.soton.acuk
}

Received Feb. 11, 2009; revised Sept. 3, 2009; accepted Sept. 15, 2009

\begin{abstract}
We consider the scattering of sound by turbulence in a jet shear layer. The turbulent, time-varying inhomogeneities in the flow scatter tonal sound fields in such a way as to give spectral broadening, which decreases the level of the incident tone, but increases the broadband level around the frequency of the tone. The scattering process is modelled for observers outside the cone of silence of the jet, using high-frequency asymptotic methods and a weak-scattering assumption. An analytical model for the far-field power spectral density of the scattered field is derived, and the result is compared to experimental data. The model correctly predicts the behaviour of the scattered field as a function of jet velocity and tone frequency.
\end{abstract}

\section{INTRODUCTION}

Spectral broadening is a phenomenon whereby a tonal sound field interacts with a random scattering medium, with the result that power is lost from the tone and distributed into a broadband field around the tone frequency. An example of the spectrum from a tonal field which has undergone spectral broadening is shown in figure 1, which is taken from the experimental work of Candel, Guedel, and Julienne [1]. The spectral broadening effect can be observed in measurements of the far-field power spectra generated by modern aeroengines operating at certain flight conditions. Typically, the effect is largest on turbine tones, though some spectral broadening of fan tones has also been observed. The effect is caused by the interaction of the tones radiated from the engine exhaust duct with the turbulence in the jet shear layer. The resulting scattered broadband field, known colloquially as a 'haystack', can be measured well above the jet-noise broadband at some engine conditions.

The modification of tone levels and the redistribution of the energy from tones into broadband noise can significantly affect the perceived noise level of the engine. It is 


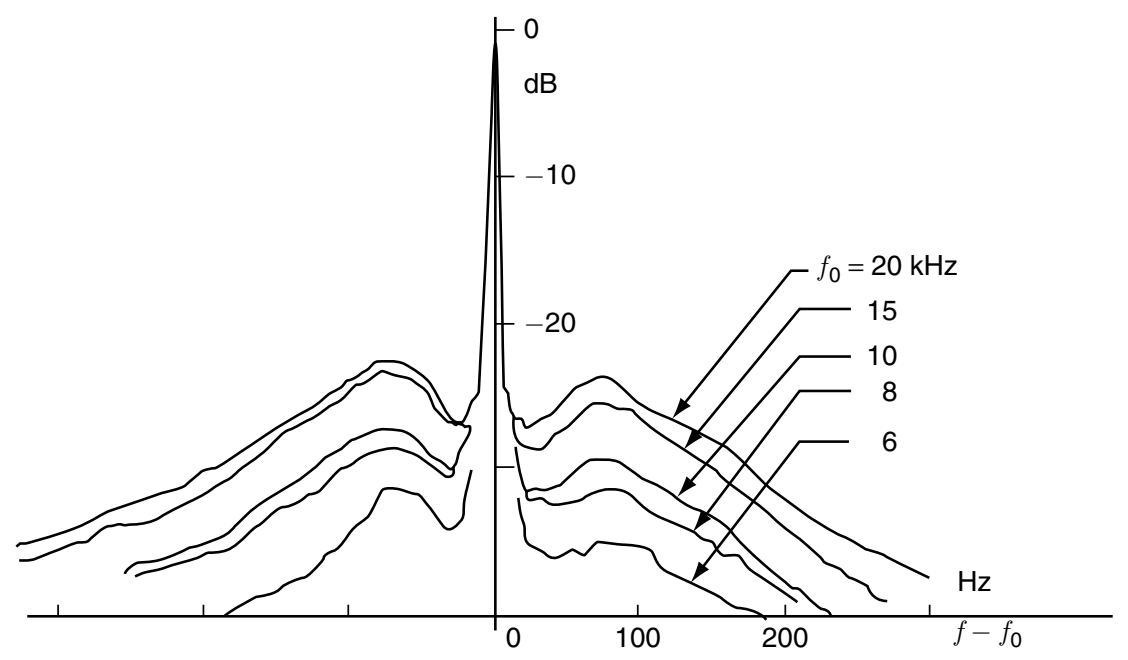

Figure 1: Spectrum of a tone having undergone 'weak' spectral broadening by a jet shear layer, for various incident frequencies $f_{0}$, measured by Candel et al [1]. (C)1975 IEEE.

possible that with sufficient understanding of the scattering process, spectral broadening could be deliberately used for noise reduction. However, a detailed analytical model of the process, in terms of the characteristics of the jet shear layer and of the turbulence, has so far been unavailable. In this paper we present such an analytical model, valid for tones radiated through a circular jet, for observers outside the cone of silence. This work is based upon a model derived by A. Cargill [2] in an internal Rolls-Royce report.

Experimental studies into acoustical haystacking in turbulent jets have been published in the public domain, but the range of results is limited. The results fall into two groups: the studies by Mathews et al [3]-[4] on the scattering of turbine tones in full-scale engine tests, and the laboratory experiments of Candel et al [1]-[5]-[6] on both small-scale jets and large wind-tunnel flows.

Mathews and Peracchio [3] carried out a series of tests on a JT3D turbofan engine with the intention of studying core and turbine noise. Their results show the presence of broadband haystacks in the spectrum, centred around the turbine tones. By comparing in-duct and far-field measurements they show that the haystack is not a product of broadband core noise, and must be a propagation effect on the turbine tones. Measurements of the spectra emitted from engines with various lengths of bypass duct fitted show that shorter bypass ducts give higher haystack levels, with correspondingly lower tone levels. This fact illustrates that the haystacking is caused by the shear layer turbulence: when a short-cowl bypass duct is fitted, the bypass shear layer has more distance upstream of the core nozzle in which to develop, so the the shear layer 'seen' by the turbine tones is thicker and more turbulent compared to the flow from a coplanar duct. The extra turbulence leads to greater haystacking, with more energy 
scattered from the tone to the haystack. Mathews, Nagel and Kester [4] give additional results to support the same conclusion.

The laboratory experiments of Candel et al [1]-[5]-[6] were designed to study the shielding effects of a jet flow, and the spectral broadening of the incident tones. Candel, Julliand and Julienne [6] placed a tonal source and a microphone array on opposite sides of a rectangular jet, and studied the blockage effect of the jet and the spectral broadening of the tones. The experimental results show that the scattered acoustic field is related to the behaviour of the turbulence. Cross-spectral analysis of the signal from two microphones reveals a time delay in the fluctuations of the scattered acoustic field: this time delay was proportional to the separation of the two microphones, and by projection of the geometry of the system was shown to be related to the convection velocity of the turbulent eddies. Correlation of the acoustic phase fluctuations with the velocity fluctuations in the shear layer (measured with a hotwire probe) offer further support for the conclusion that the spectral broadening was caused by the acoustic field interacting with the turbulence. The results also highlight the importance of the observation angle in determining the spectrum of the scattered field: at angles close to $90^{\circ}$ from the jet axis, the haystack is symmetrical about the source frequency, but away from $90^{\circ}$ the haystack becomes skewed. Candel, Guedel and Julienne [1]-[5] conducted an experiment in which a source was placed inside a circular jet and a microphone was placed outside; in this instance the dependence of the haystack on tone frequency and jet velocity was measured, but results were reported only for a microphone placed at $90^{\circ}$ to the jet axis.

The scattering of waves by turbulence is of general interest to researchers in many fields: for example, in astronomy atmospheric turbulence presents a problem for ground-based telescopes, and in underwater acoustics sonar signals are scattered by large-scale turbulence in the oceans. The general phenomenon of wave scattering by turbulent media has been examined in detail in the classic texts of Tatarski [7] and Chernov [8], whose primary interest was in the electromagnetic scattering problem. Among their results, they have shown how inhomogeneities in the propagation medium can generate random modulations of the amplitude and phase of a propagating wave.

A review of aeroacoustical work on sound propagation through turbulent flows is given by Cargill [9], who details interesting results from other fields. Cargill shows that at low frequencies, the magnitude of the scattered field is small, and the scattering is almost omnidirectional, whereas at high frequencies, the amount of scattering becomes large, and the scattered field is confined to a small angular range about the propagation direction of the incident wave. Thus in the high-frequency case, the process is essentially one of phase modulation: the sound propagates straight through the turbulence and undergoes a phase shift due to an effective fluctuation in the local refractive index.

The fact that the turbulence scattering leads to amplitude and phase modulation of the field has been used in previous analytic models of the acoustic haystacking process. A model based solely on amplitude modulation has been proposed by Hardin and Priesser [10]; however, they offer no method for relating the statistical properties of their supposed amplitude fluctuations with the physical properties of the shear layer. Experimental evidence that turbulence causes phase modulation of high-frequency 
acoustic fields is given by Schmidt and Tilmann [11]; this supports the general idea of modelling high-frequency scattering as a phase-modulating rather than an amplitude modulating process.

An analytical model for acoustic haystacking in turbulent jet flows has been developed by Campos [12]-[13], based on the idea of turbulence scattering being a phase-modulation process. The model incorporates mean-flow refraction effects, modelling the shear layer as an oscillating vortex sheet, and the convection velocity of the turbulent eddies is taken into account. Predictions made with this model match the behaviour of the measurements of Candel, Guedel and Julienne [1]-[5] at angles near $90^{\circ}$ to the jet axis. Possible problems with Campos's model are highlighted by Cargill [2], who points out that the theory is applied outside its region of validity (values are taken which are incompatible with the Rayleigh-Born hypothesis used in modelling the vortex sheet), and that at high frequencies the vortex-sheet model of the shear layer is not applicable.

An alternative analytical model for spectral broadening in jet shear layers was derived at Rolls-Royce by Cargill [2]. He applies a weak scattering theory, based on methods used in the underwater acoustics literature by, among others, Brown and Clifford [14]. In this treatment, the acoustic/turbulent interaction is modelled using an acoustic scattering equation, but the source terms in this equation require knowledge of the acoustic field. For a weak-scattering model, it is assumed that the scattered field will be of small amplitude compared to the incident field, and so one can approximate the acoustic field in the source terms by using only the incident field.

Cargill [2] begins his analysis from the point of view that the shear layer is a continuous medium through which the sound propagates, so derives a scattering equation with the Pridmore-Brown operator on the left-hand side. The mean flow is thus modelled as parallel, and refraction effects are accounted for in the propagation operator. The source terms in the scattering equation are a function of the acoustic and turbulent perturbation quantities, and the weak-scattering assumption is used to approximate the acoustic terms. The equation is then solved for the far-field power spectrum of the scattered acoustic field, using transform methods and Green's functions. The result is an analytic model which gives relationships between the turbulence properties and the scattered field, for a two-dimensional jet.

In the present paper we shall describe a new and extended version of Cargill's model. The analysis presented here is based on the same assumptions and asymptotic regimes as Cargill's, but is valid for a circular jet. The primary difference that this introduces into the analysis is the presence of scattering between azimuthal orders: an incident field consisting of a single azimuthal order will be scattered into a multi-order sound field. A secondary difference in the analysis arises in the treatment of the WKB modelling: where Cargill's model has but a single turning point, the current analysis must account for the fact that there exists a separate turning point for each order (see sections 2.2-2.3 below).

The weak-scattering model described here is useful for describing the spectral broadening in cases where the haystack peak is much lower than the tone level, as has been seen in experimental data from the papers of Mathews et al [3]-[4] and Candel et al 
[1]-[5]-[6]. In cases where the scattering is likely to be strong, an alternative model will be required, possibly based on analysis of phase-modulation by the turbulence.

An alternative method for modelling the spectral broadening is through numerical simulations. Ewert et al [15] describe computational aeroacoustic simulations in which the wave equation for sound in fluids with unsteady inhomogeneous flow is integrated. In this calculation the unsteady turbulent base flow is modelled using a stochastic method to generate turbulence features as provided by time-averaged RANS calculations. The result is a prediction of spectrum shapes which agrees with experiments.

In the current paper we shall derive an analytic weak-scattering model for the scattered field, valid for observers outside the cone of silence, and test this model against the available experimental results. First, we consider the scattering equation, and study the nature of the homogeneous solutions and Green's function of this equation. These results are then used with a weak-scattering assumption to provide the scattering source terms in the governing equation. The scattered field is then derived through the application of transform methods to the governing equation, and from this result the power spectrum in the far field is found. This result is then converted, through a stationary phase analysis, into the far-field spectral directivity. To provide validation of the model, predictions are made for the large jet studied experimentally by Candel, Guedel and Julienne [1]-[5], and it is shown that the predictions agree with the experimental results. Note that the work in this paper has been discussed in conference papers [15] and [16].

\section{DERIVATION OF MODEL}

\subsection{Governing equation and solution method}

The fluid flow in the jet is described in terms of a steady mean flow upon which a small perturbation, the sum of turbulent and acoustic fields, is superimposed. Mean flow properties are denoted with a bar, and perturbation quantities denoted with a dash. The mean flow is modelled as parallel (non-spreading) and axisymmetric. We define cylindrical coordinates $(x, r, \phi)$ such that the mean flow is in the positive $x$ direction and the mean-flow parameters are functions only of the radial coordinate $r$. The mean flow pressure, velocity, density and speed-of-sound profiles are then given by

$$
\begin{aligned}
& \bar{p}=\text { constant } \\
& \overline{\mathbf{u}}=\left(\bar{u}_{x}, \bar{u}_{r}, \bar{u}_{\phi}\right)=(U(r), 0,0), \\
& \bar{\rho}=\bar{\rho}(r), \\
& \bar{c}=\bar{c}(r)
\end{aligned}
$$

respectively. It is assumed that the flow contains a shear layer of finite width, within which the turbulence is contained, and in the limit $r \rightarrow \infty$, the mean fluid parameters 


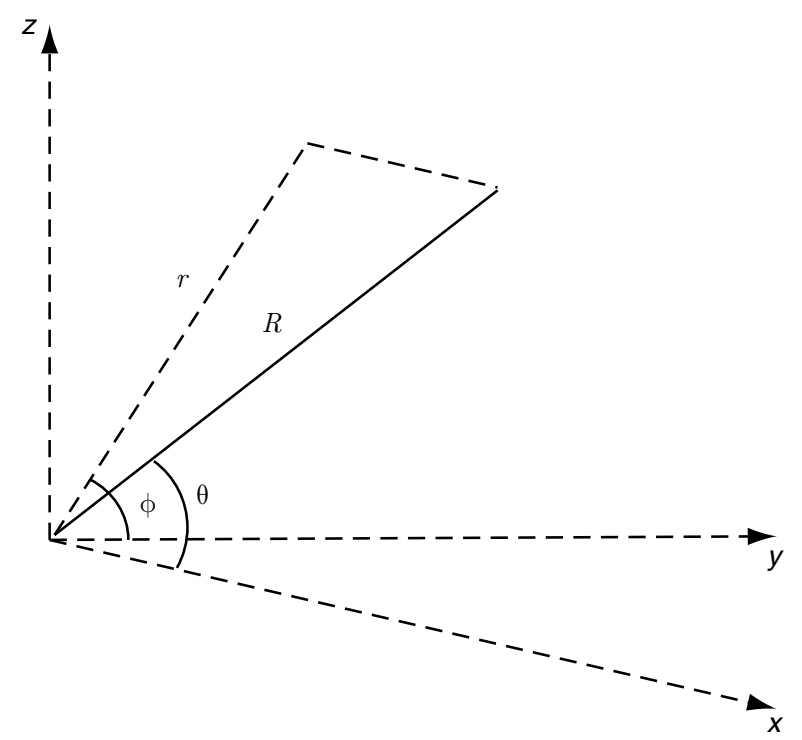

Figure 2: $\quad$ Cylidrical and spherical coordinate systems defined by equation (5).

tend to constant values, so $\bar{p} \rightarrow \bar{p}_{\infty}, U \rightarrow 0, \bar{\rho} \rightarrow \bar{\rho}_{\infty}$, and $\bar{c} \rightarrow \bar{c}_{\infty}$. In order to describe the sound in the far field, where spherical spreading is expected, we define also a spherical coordinate system $(R, \theta, \phi)$. The cylindrical and spherical coordinates are related to cartesian coordinates $\left(x_{1}, x_{2}, x_{3}\right)=(x, y, z)$ by

$$
(x, y, z)=(x, r \cos \phi, r \sin \phi)=(R \cos \theta, R \sin \theta \cos \phi, R \sin \theta \sin \phi),
$$

as shown in figure 2 .

The scattering is modelled with an approximate form of Lilley's equation, a full derivation of which for brevity is omitted here; note that a very similar version of this equation is derived by Goldstein [17] (see his equation (1.24)). In Cartesian coordinates, the scattering equation is

$$
L[p]=\frac{\overline{\mathrm{D}}}{\mathrm{D} t}\left[\bar{\rho} \frac{\partial}{\partial x_{i}}\left(\frac{F_{i}}{\bar{\rho}}\right)\right]-2 \frac{\partial \bar{u}_{i}}{\partial x_{j}} \frac{\partial F_{j}}{\partial x_{i}}-\bar{\rho} \frac{\overline{\mathrm{D}}^{2} Q}{\mathrm{D} t^{2}}
$$

where $L$ denotes the Pridmore-Brown operator (see equation (10)), and the mean-flow convected derivative operator is

$$
\frac{\overline{\mathrm{D}}}{\mathrm{D} t}=\frac{\partial}{\partial t}+\bar{U} \frac{\partial}{\partial x}
$$


The source terms $F_{i}$ and $Q$ are

$$
\begin{gathered}
F_{i}=\frac{\partial}{\partial x_{j}}\left(\bar{\rho} u_{i}^{\prime} u_{j}^{\prime}\right), \\
Q=\frac{1}{C_{p}} \frac{\partial}{\partial x_{i}}\left(s^{\prime} u_{i}^{\prime}\right),
\end{gathered}
$$

where $s^{\prime}$ is the entropy fluctuation and $C_{p}$ is the specific heat capacity at constant pressure. Note that equation (6) neglects viscous effects, and assumes that terms involving $D s / D t$ are negligible (where $s$ is the specific entropy). The perturbation terms $u_{i}^{\prime}$ and $s^{\prime}$ are the sum of acoustic and turbulent fields, and the acoustic field is itself described as the sum of two parts - the 'incident' field and the 'scattered' field. By the 'incident' field, we mean the acoustic field, say a turbine tone, which is incident on the shear layer, but described in the absence of turbulence (i.e. then 'incident' field is a solution to the homogeneous form of equation (6)). The 'scattered' field is then that field generated by the interaction of the 'incident' field with the turbulence: interaction between the scattered field and the turbulence is not considered, as we are deriving a weak scattering theory. When describing the incident and scattered fields mathematically, we shall use lower-case variables for the incident field and upper-case variables for the scattered field.

\subsection{The incident field}

In order to solve the scattering equation (6), we require solutions $p^{\prime}$ to the homogeneous equation $L\left[p^{\prime}\right]=0$, describing the incident acoustic field. In cylindrical coordinates the Pridmore-Brown operator is

$$
L=\left[\frac{\overline{\mathrm{D}}}{\mathrm{D} t}\left(\frac{1}{\bar{c}^{2}} \frac{\overline{\mathrm{D}}^{2}}{\mathrm{D} t^{2}}-\bar{\rho} \nabla \cdot\left(\frac{1}{\bar{\rho}} \nabla\right)\right)+2\left(\frac{\mathrm{d} U}{\mathrm{~d} r}\right) \frac{\partial^{2}}{\partial x \partial r}\right]
$$

In order to derive a solution to the homogeneous equation, we introduce a Fourier transform in time $t$ and the axial coordinate $x$, and a Fourier series in the azimuthal angle $\phi$. For the incident acoustic field, the transform pair is defined by

$$
\begin{gathered}
\tilde{p}_{m}^{\prime}(k, r, \omega)=\int_{-\infty}^{\infty} \int_{-\pi}^{\pi} \int_{-\infty}^{\infty} p^{\prime}(x, r, \phi, t) e^{\mathrm{i}(k x+m \phi-\omega t)} \mathrm{d} x \mathrm{~d} \phi \mathrm{d} t, \\
p^{\prime}(x, r, \phi, t)=\frac{1}{(2 \pi)^{3}} \sum_{m=-\infty}^{\infty}\left(\int_{-\infty}^{\infty} \int_{-\infty}^{\infty} \tilde{p}_{m}^{\prime}(k, r, \omega) e^{-\mathrm{i}(k x-\omega t)} \mathrm{d} k \mathrm{~d} \omega\right) e^{-\mathrm{i} m \phi},
\end{gathered}
$$


where $k, m$ and $w$ denote the axial wavenumber, azimuthal order, and frequency respectively. This transforms derivatives as follows:

$$
\frac{\partial}{\partial x} \rightarrow-\mathrm{i} k, \frac{\partial}{\partial \phi} \rightarrow-\mathrm{i} m, \frac{\partial}{\partial t} \rightarrow \mathrm{i} \omega, \text { and } \frac{\overline{\mathrm{D}}}{\mathrm{D} t} \rightarrow \mathrm{i}(\omega-k U)=\mathrm{i} d
$$

Application of the transform to the homogeneous equation leads to

$$
\frac{\bar{\rho} d^{2}}{r} \frac{\partial}{\partial r}\left(\frac{r}{\bar{\rho} d^{2}} \frac{\partial}{\partial r}\right) \tilde{p}_{m}^{\prime}+\gamma_{m}^{2} \tilde{p}_{m}^{\prime}=0
$$

where $\gamma_{m}$ is an effective wavenumber in the radial direction, given by

$$
\gamma_{m}^{2}=\frac{d^{2}}{\bar{c}^{2}}-k^{2}-\frac{m^{2}}{r^{2}} .
$$

It is assumed that a high-frequency solution (limit as $\omega \rightarrow \infty$ ) exists of the form

$$
\tilde{p}_{m}^{\prime}=A_{m}(r) \Phi_{m}\left(\xi_{m}(r)\right)
$$

where $A_{m}(r)$ is a slowly-varying amplitude function, and $\Phi_{m}\left(\xi_{m}\right)$ is a rapidly varying phase function. The amplitude function can be choosen in such a way as to simplify equation (14); specifically, we choose $A_{m}(r)$ as

$$
A_{m}(r) \propto \frac{1}{\sqrt{r}}\left(\frac{\bar{\rho} d^{2}}{\mathrm{~d} \xi_{m} / \mathrm{d} r}\right)^{1 / 2}
$$

Substitution of the solution (16) into (14) leads to

$$
\frac{\mathrm{d}^{2} \Phi_{m}}{\mathrm{~d} \xi_{m}^{2}}+\frac{\gamma_{m}^{2}}{\left(\mathrm{~d} \xi_{m} / \mathrm{d} r\right)^{2}} \Phi_{m}+\frac{\Lambda_{m}}{\left(\mathrm{~d} \xi_{m} / \mathrm{d} r\right)^{2}} \Phi_{m}=0
$$

where

$$
\Lambda_{m}=\frac{1}{A_{m}}\left(\frac{1}{r} \frac{\mathrm{d}}{\mathrm{d} r}\left(r \frac{\mathrm{d} A_{m}}{\mathrm{~d} r}\right)-\frac{\mathrm{d} A_{m}}{\mathrm{~d} r} \frac{1}{\bar{\rho} d^{2}} \frac{\mathrm{d}}{\mathrm{d} r}\left(\bar{\rho} d^{2}\right)\right) .
$$


In the high-frequency limit, $\gamma_{m}^{2}$ is $\mathrm{O}\left(\omega^{2}\right)$ as $\omega \rightarrow \infty$, so assuming that $\gamma_{m}^{2}>>\Lambda_{m}$, equation (18) reduces to

$$
\frac{\mathrm{d}^{2} \Phi_{m}}{\mathrm{~d} \xi_{m}^{2}}+\frac{\gamma_{m}^{2}}{\left(\mathrm{~d} \xi_{m} / \mathrm{d} r\right)^{2}} \Phi_{m} \approx 0 .
$$

Equation (20) is recognized as being of the form where WKB theory is applicable. However, a classical WKB solution will not be valid near a turning point, say $r=r_{m c}$, where $\gamma_{m}^{2}=0$. If $m \neq 0$, then from equation (15)

$$
\lim _{r \rightarrow 0} \gamma_{m}^{2}=-\infty \text { and } \lim _{r \rightarrow \infty} \gamma_{m}^{2}=\frac{d_{\infty}^{2}}{\bar{c}_{\infty}^{2}}-k^{2}
$$

Since $d_{\infty}=\omega$, in the high-frequency limit $\gamma_{m}^{2} \rightarrow \omega^{2} / \bar{c}_{\infty}^{2}$ as $r \rightarrow \infty$. It follows immediately that for all $m \neq 0$ there exists a turning point $r_{m_{c}} \in(0, \infty)$. To take account of this turning point, equation (20) is solved by introducing the Langer transformation, which in this case is

$$
\frac{\gamma_{m}^{2}}{\left(\mathrm{~d} \xi_{m} / \mathrm{d} r\right)^{2}}=\xi_{m}
$$

Assuming a flow profile with a single turning point at $r=r_{m c}$, then solving equation (22) leads to

$$
\begin{aligned}
& r>r_{m_{c}}, \quad \gamma_{m}^{2}>0, \quad \xi_{m}=\left(\frac{3}{2} \int_{r_{m_{c}}}^{r} \gamma_{m}\left(r^{\prime}\right) \mathrm{d} r^{\prime}\right)^{2 / 3}>0, \\
& r=r_{m_{c}}, \quad \gamma_{m}^{2}=0, \quad \xi_{m}=0 \\
& r<r_{m_{c}}, \quad \gamma_{m}^{2}<0, \quad \xi_{m}=-\left(\frac{3}{2} \int_{r}^{r_{m_{c}}}\left|\gamma_{m}\left(r^{\prime}\right)\right| \mathrm{d} r^{\prime}\right)^{2 / 3}<0,
\end{aligned}
$$

where $r^{\prime}$ is a dummy integration variable. Equation (20) then becomes

$$
\frac{\mathrm{d}^{2} \Phi_{m}}{\mathrm{~d} \xi_{m}^{2}}+\xi_{m} \Phi_{m} \approx 0
$$

which is a form of Airy's differential equation, the general solution of which is

$$
\Phi_{m}\left(\xi_{m}\right)=c_{1} \operatorname{Ai}\left(-\xi_{m}\right)+c_{2} \operatorname{Bi}\left(-\xi_{m}\right),
$$


where $\mathrm{Ai}$ and $\mathrm{Bi}$ are Airy functions, and $c_{1}$ and $c_{2}$ are arbitrary constants. The functions $\mathrm{Ai}(x)$ and $\operatorname{Bi}(x)$ are oscillatory if $x<0$, and non-oscillatory if $x>0$. Application of the radiation condition as $r \rightarrow \infty$ leads to $c_{1}=-\mathrm{i} c_{2}$, so

$$
\Phi_{m}\left(\xi_{m}\right)=c_{2}\left(\operatorname{Bi}\left(-\xi_{m}\right)-\mathrm{iAi}\left(-\xi_{m}\right)\right)
$$

Then, as $r \rightarrow \infty$ (and $\xi_{m} \rightarrow \infty$ ), for observers outside the cone of silence,

$$
\tilde{p}_{m}^{\prime} \sim \frac{c_{2}}{\sqrt{r}}\left(\frac{\bar{\rho}_{\infty} d_{\infty}^{2}}{\gamma_{m_{\infty}}}\right)^{1 / 2} \frac{1}{\sqrt{\pi}} e^{-\mathrm{i} \pi / 4} \exp \left\{-\mathrm{i} \int_{r_{m_{e}}}^{r} \gamma_{m}\left(r^{\prime}\right) \mathrm{d} r^{\prime}\right\}
$$

A far-field transformed pressure is defined as

$$
\tilde{p}_{m_{\infty}}^{\prime}(k, \omega)=\lim _{r \rightarrow \infty} \sqrt{r} \tilde{p}_{m}^{\prime}(k, r, \omega) \exp \left\{\mathrm{i} \int_{r_{m_{c}}}^{r} \gamma_{m}\left(r^{\prime}\right) \mathrm{d} r^{\prime}\right\}
$$

evaluation of the limit, with equation (27), leads to an expression for $c_{2}$ :

$$
c_{2}=\tilde{p}_{m_{\infty}}^{\prime}(k, \omega)\left(\frac{\gamma_{m_{\infty}}}{\bar{\rho}_{\infty} d_{\infty}^{2}}\right)^{1 / 2} \sqrt{\pi} e^{\mathrm{i} \pi / 4}
$$

Substitution of this value for $c_{2}$ into equation (26) leads to

$$
\tilde{p}_{m}^{\prime}(k, r, \omega)=\tilde{p}_{m_{\infty}}^{\prime}(k, \omega) \sqrt{\frac{\pi}{r}} e^{\mathrm{i} \pi / 4}\left(\frac{\bar{\rho}}{\bar{\rho}_{\infty}}\right)^{1 / 2}\left(\frac{d}{d_{\infty}}\right)\left(\frac{\gamma_{m_{\infty}}}{\gamma_{m}}\right)^{1 / 2} \xi_{m}^{1 / 4}\left[\operatorname{Bi}\left(-\xi_{m}\right)-\mathrm{iAi}\left(-\xi_{m}\right)\right]
$$

In the current analysis, we shall derive a solution for observers situated outside the cone of silence. In this case, the scattering points are likely to be well above the turning points of the WKB solution, so $\xi_{m}$ is large and positive; the pressure field given by equation (30) then reduces to the asymptotic form

$$
\tilde{p}_{m}^{\prime}(k, r, \omega) \approx \tilde{p}_{m_{\infty}}^{\prime}(k, \omega) \sqrt{\frac{1}{r}}\left(\frac{\bar{\rho}}{\bar{\rho}_{\infty}}\right)^{1 / 2}\left(\frac{d}{d_{\infty}}\right)\left(\frac{\gamma_{m_{\infty}}}{\gamma_{m}}\right)^{1 / 2} \exp \left\{-\mathrm{i} \int_{r_{m_{c}}}^{r} \gamma_{m}\left(r^{\prime}\right) \mathrm{d} r^{\prime}\right\} .
$$




\subsection{The Green's function}

To derive the scattered field, we require a Green's function $G$ which describes radiation from a point source: this function is a solution to the equation

$$
L[G]=\delta(t) \delta\left(x-x_{0}\right) \frac{\delta\left(r-r_{0}\right)}{r} \sum_{n=-\infty}^{\infty} \delta\left(\phi-\phi_{0}-2 \pi n\right) .
$$

The required Green's function, and its relation to geometrical acoustics, has been discussed in detail by Wundrow and Khavaran [18]. Since the jet is parallel and axisymmetric, we can without loss of generality set $\mathbf{x}_{0}=\left(x_{0}, r_{0}, \phi_{0}\right)=\left(0, r_{0}, 0\right)$. To solve this equation, we define a Fourier transform as above; however, we note that to describe the scattered field we shall use upper-case variables, where for the incident field we used lower-case variables. Thus the transform pair in this case is

$$
\begin{gathered}
\tilde{G}_{M}(K, r, \Omega)=\int_{-\infty}^{\infty} \int_{-\pi}^{\pi} \int_{-\infty}^{\infty} G(x, r, \phi, t) e^{\mathrm{i}(K x+M \phi-\Omega t)} \mathrm{d} x \mathrm{~d} \phi \mathrm{d} t, \\
G(x, r, \phi, t)=\frac{1}{(2 \pi)^{3}} \sum_{M=-\infty}^{\infty}\left(\int_{-\infty}^{\infty} \int_{-\infty}^{\infty} \tilde{G}_{M}(K, r, \Omega) e^{-\mathrm{i}(K x-\Omega t)} \mathrm{d} K \mathrm{~d} \Omega\right) e^{-\mathrm{i} M \phi},
\end{gathered}
$$

where $K, M$ and $\Omega$ denote the axial wavenumber, azimuthal order, and frequency respectively. This transforms derivatives as follows:

$$
\frac{\partial}{\partial x} \rightarrow-\mathrm{i} K, \frac{\partial}{\partial \phi} \rightarrow-\mathrm{i} M, \frac{\partial}{\partial t} \rightarrow \mathrm{i} \Omega, \text { and } \frac{\overline{\mathrm{D}}}{\mathrm{D} t} \rightarrow \mathrm{i}(\Omega-K U)=\mathrm{i} D
$$

Application of the transform to equation (32) leads to

$$
\frac{\bar{\rho} D^{2}}{r} \frac{\partial}{\partial r}\left(\frac{r}{\bar{\rho} D^{2}} \frac{\partial}{\partial r}\right) \tilde{G}_{M}+\Gamma_{M}^{2} \tilde{G}_{M}=\frac{\mathrm{i}}{D_{0}} \frac{\delta\left(r-r_{0}\right)}{r_{0}}
$$

where $\Gamma_{M}$ is an effective wavenumber in the radial direction,

$$
\Gamma_{M}^{2}=\frac{D^{2}}{\bar{c}^{2}}-K^{2}-\frac{M^{2}}{r^{2}}
$$

and subscript 0 denotes a value at the source position $\mathbf{x}_{0}$.

The Green's function can be derived by the same method as that applied above for the incident field. Application of the high-frequency approximations and the Langer 
transformation allows the solution to be written in terms of Airy functions, and with a radiation condition at $r \rightarrow \infty$ and a finiteness condition at $r \rightarrow 0$ it can be shown that

$$
\begin{aligned}
\tilde{G}_{M}\left(r \mid r_{0}\right)= & \left(\frac{\pi}{\mathrm{i} D_{0}}\right)\left(\frac{1}{\sqrt{r} \sqrt{r_{0}}}\right)\left(\frac{\bar{\rho}}{\bar{\rho}_{0}}\right)^{1 / 2}\left(\frac{D}{D_{0}}\right)\left(\frac{\zeta_{M}}{\Gamma_{M}^{2}}\right)^{1 / 4}\left(\frac{\zeta_{M_{0}}}{\Gamma_{M_{0}}^{2}}\right)^{1 / 4} \\
& \times\left[\operatorname{Bi}\left(-\zeta_{M>}\right)-\mathrm{iAi}\left(-\zeta_{M>}\right)\right] \operatorname{Ai}\left(-\zeta_{M<}\right) .
\end{aligned}
$$

Here $\zeta_{M>}$ denotes $\zeta_{M}$ for $r>r_{0}$ and $\zeta_{M_{0}}$ for $r<r_{0}$; likewise $\zeta_{M<}$ denotes $\zeta_{M_{0}}$ for $r>r_{0}$ and $\zeta_{M}$ for $r<r_{0}$. Note that $\zeta_{M}$ in this case behaves similarly to $\xi_{M}$ in equation (23). For observers outside the cone of silence in the far field, and for scatterers above the turning point (i.e. $\zeta_{M_{0}}$ large and positive), this solution can be written in the approximate form

$$
\begin{aligned}
\tilde{G}_{M}\left(r \mid r_{0}\right) \approx & \left.\frac{1}{-2 D_{0}}\right)\left(\frac{1}{\sqrt{r} \sqrt{r_{0}}}\right)\left(\frac{\bar{\rho}_{\infty}}{\bar{\rho}_{0}}\right)^{1 / 2}\left(\frac{D_{\infty}}{D_{0}}\right)\left(\frac{1}{\Gamma_{M_{\infty}}^{2}}\right)^{1 / 4}\left(\frac{1}{\Gamma_{M_{0}}^{2}}\right)^{1 / 4} \\
& \times \exp \left[-\mathrm{i} \int_{r_{0}}^{r} \Gamma_{M}\left(r^{\prime}\right) \mathrm{d} r^{\prime}\right] X\left(r_{0}\right) .
\end{aligned}
$$

The function $X\left(r_{0}\right)$ is given by

$$
X\left(r_{0}\right)=1+\mathrm{e}^{\mathrm{i} \pi / 2} \exp \left[-\mathrm{i} 2 \int_{r_{M_{c}}}^{r_{0}} \Gamma_{M}\left(r^{\prime}\right) \mathrm{d} r^{\prime}\right],
$$

where $r_{M_{c}}$ denotes the turning point; this function describes the fact that for the given source and observer, there are two possible ray paths between source and observer. The first term in $X\left(r_{0}\right)$ describes the direct path to the far field, while the second term describes a ray radiated down towards the jet centreline and then reflected at the turning point: these paths are sketched in figure 3. The solution (39) describes the Green's function, composed of coherent contributions from two ray paths, derived for a steady mean flow. In the presence of turbulence (both in the shear layer and atmospheric) we expect that the two rays will not sum coherently: for this reason, we omit the reflected ray (ray 2 in figure 3 ) in the Green's function, by letting $X\left(r_{0}\right)=1$. This assumption will simplify the following analysis, albeit the predicted far-field sound power level will be $3 \mathrm{~dB}$ lower compared to adding the direct and indirect rays incoherently.

At high frequency the derivatives of the Green's function (39) are approximated as

$$
\frac{\partial \tilde{G}_{M}}{\partial r_{0}} \approx \mathrm{i} \Gamma_{M_{0}} \tilde{G}_{M}, \text { and } \frac{\partial^{2} \tilde{G}_{M}}{\partial r_{0}^{2}} \approx-\Gamma_{M_{0}}^{2} \tilde{G}_{M} .
$$




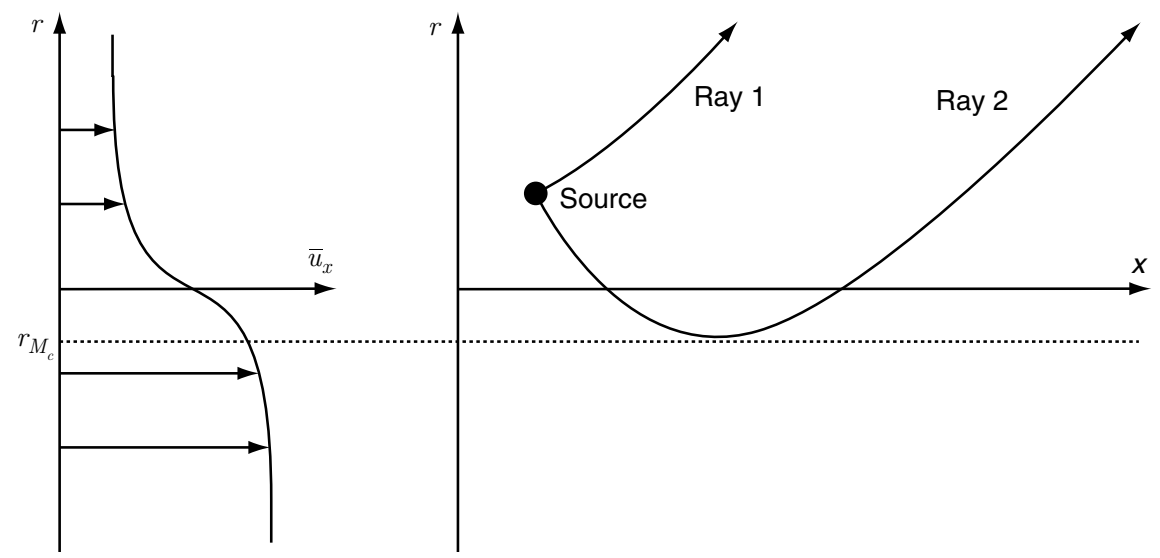

Figure 3: Two rays propagate from the source towards the same far-field point. Left: velocity profile with turning point $r_{M_{c}}$ Right: rays paths. Ray 1 propagates straight to the far field, while ray 2 is reflected at the turning point.

\subsection{Modelling source terms}

The solution of the scattering equation is derived using a high-frequency approximation, which means that source terms involving gradients of mean-flow quantities can be neglected. Thus, the source terms to be utilized in this analysis reduce to

$$
\bar{\rho} \frac{\overline{\mathrm{D}}}{\mathrm{D} t}\left[\frac{\partial^{2}}{\partial x_{i} \partial x_{k}}\left(u_{i}^{\prime} u_{k}^{\prime}\right)\right]-\frac{\bar{\rho}}{C_{p}} \frac{\overline{\mathrm{D}}^{2}}{\mathrm{D} t^{2}}\left[\frac{\partial}{\partial x_{i}}\left(s^{\prime} u_{i}^{\prime}\right)\right] .
$$

These source terms may be expressed in cylindrical polar coordinates, as

$$
\begin{aligned}
\bar{\rho} \frac{\overline{\mathrm{D}}}{\mathrm{D} t}[ & \frac{\partial^{2}}{\partial x^{2}}\left(u_{x}^{\prime} u_{x}^{\prime}\right)+\frac{\partial^{2}}{\partial x \partial r}\left(u_{x}^{\prime} u_{r}^{\prime}\right)+\frac{1}{r} \frac{\partial}{\partial x}\left(u_{x}^{\prime} u_{r}^{\prime}\right)+\frac{1}{r} \frac{\partial^{2}}{\partial x \partial \phi}\left(u_{x}^{\prime} u_{\phi}^{\prime}\right)+\frac{\partial^{2}}{\partial r \partial x}\left(u_{r}^{\prime} u_{x}^{\prime}\right) \\
& +\frac{1}{r} \frac{\partial}{\partial x}\left(u_{r}^{\prime} u_{x}^{\prime}\right)+\frac{\partial^{2}}{\partial r^{2}}\left(u_{r}^{\prime} u_{r}^{\prime}\right)+\frac{2}{r} \frac{\partial}{\partial r}\left(u_{r}^{\prime} u_{r}^{\prime}\right)+\frac{1}{r} \frac{\partial^{2}}{\partial r \partial \phi}\left(u_{r}^{\prime} u_{\phi}^{\prime}\right)+\frac{1}{r^{2}} \frac{\partial}{\partial \phi}\left(u_{r}^{\prime} u_{\phi}^{\prime}\right) \\
& \left.+\frac{1}{r} \frac{\partial^{2}}{\partial \phi \partial x}\left(u_{\phi}^{\prime} u_{x}^{\prime}\right)+\frac{1}{r} \frac{\partial^{2}}{\partial \phi \partial r}\left(u_{\phi}^{\prime} u_{r}^{\prime}\right)+\frac{1}{r^{2}} \frac{\partial}{\partial \phi}\left(u_{\phi}^{\prime} u_{r}^{\prime}\right)+\frac{1}{r^{2}} \frac{\partial^{2}}{\partial \phi^{2}}\left(u_{\phi}^{\prime} u_{\phi}^{\prime}\right)-\frac{1}{r} \frac{\partial}{\partial r}\left(u_{\phi}^{\prime} u_{\phi}^{\prime}\right)\right] \\
& -\frac{\bar{\rho}}{C_{p}} \frac{\overline{\mathrm{D}}^{2}}{\mathrm{D} t^{2}}\left[\frac{\partial}{\partial x}\left(s^{\prime} u_{x}^{\prime}\right)+\frac{\partial}{\partial r}\left(s^{\prime} u_{r}^{\prime}\right)+\frac{1}{r}\left(s^{\prime} u_{r}^{\prime}\right)+\frac{1}{r} \frac{\partial}{\partial \phi}\left(s^{\prime} u_{\phi}^{\prime}\right)\right] .
\end{aligned}
$$

The double divergence term $\partial^{2}\left(u_{i}^{\prime} u_{k}^{\prime}\right) / \partial x_{i} \partial x_{k}$, expressed in cylindrical polar coordinates, was derived originally by Tester and Morfey [19]. 
The source terms (43) are written simply in terms of the pertubation quantities $\mathbf{u}^{\prime}$ and $s^{\prime}$. These perturbations are each the sum of a turbulent and an acoustic field, which we denote by subscripts $t$ for turbulence and $s$ for sound. Since we are interested in the scattering of the acoustic field by the turbulence, rather than the jet noise for example, we shall study only those terms which contain acoustic and turbulent contributions. Thus

$$
\begin{aligned}
u_{i}^{\prime} u_{j}^{\prime} & =\left(u_{i t}^{\prime}+u_{i s}^{\prime}\right)\left(u_{j t}^{\prime}+u_{j s}^{\prime}\right), \\
& =u_{i t}^{\prime} u_{j t}^{\prime}+u_{i t}^{\prime} u_{j s}^{\prime}+u_{i s}^{\prime} u_{j t}^{\prime}+u_{i s}^{\prime} u_{j s}^{\prime}, \\
& \approx u_{i t}^{\prime} u_{j s}^{\prime}+u_{i s}^{\prime} u_{j t}^{\prime}, \\
s^{\prime} u_{i}^{\prime} & =\left(s_{t}^{\prime}+s_{s}^{\prime}\right)\left(u_{i t}^{\prime}+u_{i s}^{\prime}\right), \\
& =s_{t}^{\prime} u_{i t}^{\prime}+s_{t}^{\prime} u_{i s}^{\prime}+s_{s}^{\prime} u_{i t}^{\prime}+s_{s}^{\prime} u_{i s}^{\prime}, \\
& \approx s_{t}^{\prime} u_{i s}^{\prime},
\end{aligned}
$$

where subscripts $i$ and $j$ denote $x, r$ or $\phi$. The acoustic entropy fluctuation $s_{s}^{\prime}$ is assumed to be negligible.

In order to solve the scattering equation with the above source distribution, we require the acoustic velocity field $\mathbf{u}_{s}^{\prime}$. This is derived using the weak-scattering assumption: if the magnitude of the scattered acoustic field is small compared to that of the incident acoustic field, then $\mathbf{u}_{s}^{\prime}$ is approximated using only the incident field (31). The velocity is related to the pressure through the linearised momentum equation

$$
\bar{\rho} \frac{\overline{\mathrm{D}}}{\mathrm{D} t} u_{i s}^{\prime}=-\nabla p_{s}^{\prime}
$$

which in the incident field transform domain defined by equations (11)-(12) is

$$
\tilde{u}_{i s_{m}}^{\prime}(k, r, \omega)=\frac{1}{\bar{\rho} d}\left(k, \mathrm{i} \frac{\partial}{\partial r}, \frac{m}{r}\right)_{i} \tilde{p}_{s_{m}}^{\prime}(k, r, \omega) .
$$

In the high-frequency limit, this transformed incident velocity can be approximated as

$$
\tilde{u}_{i s_{m}}^{\prime}(k, r, \omega)=\frac{1}{\bar{\rho} d}\left(k, \gamma_{m}, \frac{m}{r}\right)_{i} \tilde{p}_{s_{m}}^{\prime}(k, r, \omega) .
$$

\subsection{The scattered pressure field}

The source terms in the scattering equation (6) are now, in principle, known quantites, so we may proceed to solve the equation for the scattered acoustic field $P^{\prime}(x, r, \phi, t)$. 
Application of the scattered field Fourier transform (33)-(34), denoted here with an $\mathcal{F}$ operator, to the scattering equation (6) with approximate source distribution (43), leads to

$$
-\mathrm{i} D\left[\frac{\bar{\rho} D^{2}}{r} \frac{\partial}{\partial r}\left(\frac{r}{\bar{\rho} D^{2}} \frac{\partial}{\partial r}\right) \tilde{P}_{M}^{\prime}+\Gamma_{M}^{2} \tilde{P}_{M}^{\prime}\right] \approx \tilde{S}_{M}(r),
$$

where $\widetilde{S}_{M}(r)$ denotes the $M$ th azimuthal mode of the transformed source distribution:

$$
\begin{aligned}
\tilde{S}_{M}(r)=\mathrm{i} \bar{\rho} D( & -K^{2} \mathcal{F}\left\{u_{x}^{\prime} u_{x}^{\prime}\right\}-2 \mathrm{i} K \frac{\mathrm{d}}{\mathrm{d} r} \mathcal{F}\left\{u_{r}^{\prime} u_{x}^{\prime}\right\}-\frac{2 \mathrm{i} K}{r} \mathcal{F}\left\{u_{r}^{\prime} u_{x}^{\prime}\right\}-\frac{2 M K}{r} \mathcal{F}\left\{u_{\phi}^{\prime} u_{x}^{\prime}\right\} \\
& +\frac{\mathrm{d}^{2}}{\mathrm{~d} r^{2}} \mathcal{F}\left\{u_{r}^{\prime} u_{r}^{\prime}\right\}+\frac{2}{r} \frac{\mathrm{d}}{\mathrm{d} r} \mathcal{F}\left\{u_{r}^{\prime} u_{r}^{\prime}\right\}-\frac{2 \mathrm{i} M}{r} \frac{\mathrm{d}}{\mathrm{d} r} \mathcal{F}\left\{u_{r}^{\prime} u_{\phi}^{\prime}\right\}-\frac{2 \mathrm{i} M}{r^{2}} \mathcal{F}\left\{u_{r}^{\prime} u_{\phi}^{\prime}\right\} \\
& \left.-\frac{M^{2}}{r^{2}} \mathcal{F}\left\{u_{\phi}^{\prime} u_{\phi}^{\prime}\right\}-\frac{1}{r} \frac{\mathrm{d}}{\mathrm{d} r} \mathcal{F}\left\{u_{\phi}^{\prime} u_{\phi}^{\prime}\right\}\right)+\frac{\bar{\rho} D^{2}}{C_{p}}\left(-\mathrm{i} K \mathcal{F}\left\{s^{\prime} u_{x}^{\prime}\right\}+\frac{\mathrm{d}}{\mathrm{d} r} \mathcal{F}\left\{s^{\prime} u_{r}^{\prime}\right\}\right. \\
& \left.+\frac{1}{r} \mathcal{F}\left\{s^{\prime} u_{r}^{\prime}\right\}-\frac{\mathrm{i} M}{r} \mathcal{F}\left\{s^{\prime} u_{\phi}^{\prime}\right\}\right) .
\end{aligned}
$$

The solution to this transformed equation (49) can be found using a Green's function, with the general result

$$
\tilde{P}_{M}^{\prime}=\int_{r_{0}=0}^{\infty} \tilde{G}_{M}\left(r \mid r_{0}\right) \tilde{S}_{M}\left(r_{0}\right) r_{0} \mathrm{~d} r_{0}
$$

Expressing the perturbation quantities in $\widetilde{S}$ in terms of turbulent and acoustic parts, as in equations (44) and (45) leads to

$$
\begin{aligned}
\tilde{P}_{M}^{\prime}(K, r, \Omega)= & \int_{r_{0}=0}^{\infty} \tilde{G}_{M}\left(r \mid r_{0}\right)\left[\mathrm { i } \overline { \rho } _ { 0 } D _ { 0 } \left(-2 K^{2} \mathcal{F}_{0}\left\{u_{x t}^{\prime} u_{x s}^{\prime}\right\}-2 \mathrm{i} K \frac{\mathrm{d}}{\mathrm{d} r_{0}} \mathcal{F}_{0}\left\{u_{x t}^{\prime} u_{r s}^{\prime}\right\}\right.\right. \\
& -2 \mathrm{i} K \frac{\mathrm{d}}{\mathrm{d} r_{0}} \mathcal{F}_{0}\left\{u_{r t}^{\prime} u_{x s}^{\prime}\right\}-\frac{2 \mathrm{i} K}{r_{0}} \mathcal{F}_{0}\left\{u_{x t}^{\prime} u_{r s}^{\prime}\right\}-\frac{2 \mathrm{i} K}{r_{0}} \mathcal{F}_{0}\left\{u_{r t}^{\prime} u_{x s}^{\prime}\right\} \\
& -\frac{2 M K}{r_{0}} \mathcal{F}_{0}\left\{u_{x t}^{\prime} u_{\phi s}^{\prime}\right\}-\frac{2 M K}{r_{0}} \mathcal{F}_{0}\left\{u_{\phi t}^{\prime} u_{x s}^{\prime}\right\}+2 \frac{\mathrm{d}^{2}}{\mathrm{~d} r_{0}^{2}} \mathcal{F}_{0}\left\{u_{r t}^{\prime} u_{r s}^{\prime}\right\} \\
& +\frac{4}{r_{0}} \frac{\mathrm{d}}{\mathrm{d} r_{0}} \mathcal{F}_{0}\left\{u_{r t}^{\prime} u_{r s}^{\prime}\right\}-\frac{2 \mathrm{i} M}{r_{0}} \frac{\mathrm{d}}{\mathrm{d} r_{0}} \mathcal{F}_{0}\left\{u_{r t}^{\prime} u_{\phi s}^{\prime}\right\}-\frac{2 \mathrm{i} M}{r_{0}} \frac{\mathrm{d}}{\mathrm{d} r_{0}} \mathcal{F}_{0}\left\{u_{\phi t}^{\prime} u_{r s}^{\prime}\right\} \\
& -\frac{2 \mathrm{i} M}{r_{0}^{2}} \mathcal{F}_{0}\left\{u_{r t}^{\prime} u_{\phi s}^{\prime}\right\}-\frac{2 \mathrm{i} M}{r_{0}^{2}} \mathcal{F}_{0}\left\{u_{\phi t}^{\prime} u_{r s}^{\prime}\right\}-\frac{2 M^{2}}{r_{0}^{2}} \mathcal{F}_{0}\left\{u_{\phi t}^{\prime} u_{\phi s}^{\prime}\right\}
\end{aligned}
$$




$$
\begin{aligned}
& \left.-\frac{2}{r_{0}} \frac{\mathrm{d}}{\mathrm{d} r_{0}} \mathcal{F}_{0}\left\{u_{\phi t}^{\prime} u_{\phi s}^{\prime}\right\}\right)+\frac{\bar{\rho}_{0} D_{0}^{2}}{C_{p}}\left(-\mathrm{i} K \mathcal{F}_{0}\left\{s_{t}^{\prime} u_{x s}^{\prime}\right\}+\frac{\mathrm{d}}{\mathrm{d} r_{0}} \mathcal{F}_{0}\left\{s_{t}^{\prime} u_{r s}^{\prime}\right\}\right. \\
& \left.\left.+\frac{1}{r_{0}} \mathcal{F}_{0}\left\{s_{t}^{\prime} u_{r s}^{\prime}\right\}-\frac{\mathrm{i} M}{r_{0}} \mathcal{F}_{0}\left\{s_{t}^{\prime} u_{\phi s}^{\prime}\right\}\right)\right] r_{0} d r_{0},
\end{aligned}
$$

where $\mathcal{F}_{0}\{\}$ denotes $\left.\mathcal{F}\{\}\right|_{r=r_{0}}$.

Equation (52) involves radial derivatives of unknown turbulence functions, but it is possible, through integration by parts, to make these derivatives act on the Green's function, whose derivatives are given by equation (41). For example,

$$
\begin{aligned}
\int_{r_{0}=0}^{\infty} \tilde{G}_{M}\left(r \mid r_{0}\right) \frac{\mathrm{d}}{\mathrm{d} r_{0}} \mathcal{F}_{0}\{\Psi\} \mathrm{d} r_{0} & =-\int_{r_{0}=0}^{\infty} \frac{\mathrm{d}}{\mathrm{d} r_{0}}\left(\tilde{G}_{M}\left(r \mid r_{0}\right)\right) \mathcal{F}_{0}\{\Psi\} \mathrm{d} r_{0} \\
& \approx-\mathrm{i} \int_{r_{0}=0}^{\infty} \Gamma_{M_{0}} \tilde{G}_{M}\left(r \mid r_{0}\right) \mathcal{F}_{0}\{\Psi\} \mathrm{d} r_{0}
\end{aligned}
$$

assuming that the turbulent quantities equal zero at $r=0$ and $r \rightarrow \infty$. Further, equation (52) contains many terms which are Fourier transforms of products of perturbation quantities: such terms can be written in terms of the transforms of the individual perturbations by application of a convolution. For the velocity terms this is

$$
\mathcal{F}_{0}\left\{u_{i t}^{\prime} u_{j s}^{\prime}\right\}=\frac{1}{(2 \pi)^{3}} \sum_{m=-\infty}^{\infty} \int_{-\infty}^{\infty} \int_{-\infty}^{\infty} u_{i t_{M-m}}^{\prime}\left(K-k, r_{0}, \Omega-\omega\right) u_{j s_{m}}^{\prime}\left(k, r_{0}, \omega\right) \mathrm{d} k \mathrm{~d} \omega
$$

the expression for entropy terms is similar. Note that the products of turbulent and acoustic quantities are ordered such that all acoustic quantities are evaluated at the incident values $\left(k, r_{0}, \omega\right)$, and turbulent quantities are evaluated at difference values $\left(K-k, r_{0}, \Omega-\omega\right)$. Finally, we recall that the acoustic velocity perturbation terms can be written in terms of the incident pressure field, as in equation (48).

Upon application of the integration by parts, convolutions, and substitution discussed, equation (52) becomes

$$
\begin{aligned}
P_{M}^{\prime}(K, r, \Omega)= & \frac{1}{(2 \pi)^{3}} \sum_{m=-\infty}^{\infty} \int_{\omega=-\infty}^{\infty} \int_{k=-\infty}^{\infty} \int_{r_{0}=0}^{\infty}-2 \mathrm{i} \frac{D_{0}^{2}}{d_{0}} r_{0} G_{M}\left(r \mid r_{0}\right) p_{m_{0}}^{\prime} \times \\
& {\left[( K k + \Gamma _ { M _ { 0 } } \gamma _ { m _ { 0 } } + \frac { M m } { r _ { 0 } ^ { 2 } } ) \left(\frac{K}{D_{0}} u_{x t_{M-m}}^{\prime}+\frac{\Gamma_{M_{0}}}{D_{0}} u_{r t_{M-m}}^{\prime}+\frac{M / r_{0}}{D_{0}} u_{\phi t_{M-m}}^{\prime}\right.\right.}
\end{aligned}
$$




$$
\begin{aligned}
& +\frac{\mathrm{i}}{r_{0} D_{0}}\left(\Gamma_{M_{0}} \gamma_{m_{0}}+\frac{M m}{r_{0}^{2}}\right) \tilde{u}_{r t_{M-m}}^{\prime}+\frac{i}{r_{0} D_{0}}\left(\frac{M}{r_{0}} \gamma_{m_{0}}-\frac{m}{r_{0}} \Gamma_{M_{0}}\right) \tilde{u}_{\phi t_{M-m}}^{\prime} \\
& \left.+\left(K k+\Gamma_{M_{0}} \gamma_{m_{0}}+\frac{M m}{r_{0}^{2}}\right) \frac{1}{2 C_{p}} \tilde{s}_{t_{M-m}}^{\prime}\right] \mathrm{d} r_{0} \mathrm{~d} k \mathrm{~d} \omega .
\end{aligned}
$$

In the high frequency limit, two of the terms in the square brackets in (56) can be neglected, as they are small compared to the remaining terms. Then

$$
\begin{aligned}
P_{M}^{\prime}(K, r, \Omega) \approx & \frac{1}{(2 \pi)^{3}} \sum_{m=-\infty}^{\infty} \int_{\omega=-\infty}^{\infty} \int_{k=-\infty}^{\infty} \int_{r_{0}=0}^{\infty}-2 \mathrm{i} \frac{D_{0}^{2}}{d_{0}} r_{0} G_{M}\left(r \mid r_{0}\right) p_{m_{0}}^{\prime} \Lambda\left(r_{0}\right) \times \\
& \left(\frac{K}{D_{0}} u_{x t_{M-m}}^{\prime}+\frac{\Gamma_{M_{0}}}{D_{0}} u_{r t_{M-m}}^{\prime}+\frac{M / r_{0}}{D_{0}} u_{\phi t_{M-m}}^{\prime}+\frac{1}{2 C_{p}} s_{t_{M-m}}^{\prime}\right) \mathrm{d} r_{0} \mathrm{~d} k \mathrm{~d} \omega,
\end{aligned}
$$

where expressions for $\tilde{p}_{m_{0}}^{\prime}$ and $\widetilde{G}_{M}\left(r \mid r_{0}\right)$ are given by equations (31) and (39) respectively, and we define

$$
\Lambda\left(r_{0}\right)=K k+\Gamma_{M_{0}} \gamma_{m_{0}}+\frac{M m}{r_{0}^{2}}
$$

Evaluation of equation (57) requires knowledge of the turbulent velocity and entropy fluctuations. In general, we only know the statistical properties of these fluctuations, which we consider below.

\subsection{Turbulence correlation functions}

The turbulent velocity and entropy cross-correlation functions are defined as

$$
\begin{aligned}
& R_{i j}=\left\langle u_{i t}^{\prime}\left(x_{1}, r_{01}, \phi_{1}, t_{1}\right) u_{j t}^{\prime \star}\left(x_{2}, r_{02}, \phi_{2}, t_{2}\right)\right\rangle, \\
& R_{s}=\left\langle s_{t}^{\prime}\left(x_{1}, r_{01}, \phi_{1}, t_{1}\right) s_{t}^{\prime \star}\left(x_{2}, r_{02}, \phi_{2}, t_{2}\right)\right\rangle,
\end{aligned}
$$

respectively, where \langle\rangle denotes the ensemble average, and ${ }^{\star}$ denotes the complex conjugate. Note that we assume the velocity and entropy fluctuations are uncorrelated with one another, so

$$
\left\langle u_{i t}^{\prime} s_{t}^{\prime *}\right\rangle=0
$$


Since the mean flow is steady, parallel, and axisymmetric, we assume that the turbulence is statistically stationary in the variables $x, \phi$, and $t$. Then $R_{i j}$ and $R_{s}$ are functions only of the separation variables

$$
\mu_{x}=x_{1}-x_{2}, \mu_{r}=r_{01}-r_{02}, \mu_{\phi}=\phi_{1}-\phi_{2}, \tau=t_{1}-t_{2}
$$

and the mean radial position variable

$$
\bar{r}=\frac{1}{2}\left(r_{01}+r_{02}\right) .
$$

A correlation function of the transformed turbulent velocity can be defined, and it can be shown that

$$
\begin{aligned}
& \left\langle\tilde{u}_{i t_{M-m_{1}}}^{\prime}\left(K-k_{1}, r_{01}, \Omega-\omega_{1}\right) \tilde{u}_{j t_{M-m_{2}}}^{\prime \star}\left(K-k_{2}, r_{02}, \Omega-\omega_{2}\right)\right\rangle= \\
& \quad(2 \pi)^{3} \delta\left(k_{1}-k_{2}\right) \delta\left(m_{1}-m_{2}\right) \delta\left(\omega_{2}-\omega_{1}\right) \times \\
& \quad \int_{\mu_{x}=-\infty}^{\infty} 2 \int_{\mu_{\phi}=-\pi}^{\pi} \int_{\tau=-\infty}^{\infty} R_{i j} \mathrm{e}^{\mathrm{i}\left(K-\left(k_{1}+k_{2}\right) / 2\right) \mu_{x}} \mathrm{e}^{\mathrm{i}\left(M-\left(m_{1}+m_{2}\right) / 2\right) \mu_{\phi}} \mathrm{e}^{-\mathrm{i}\left(\Omega-\left(\omega_{1}+\omega_{2}\right) / 2\right) \tau} \mathrm{d} \tau \mathrm{d} \mu_{\phi} \mathrm{d} \mu_{x} .
\end{aligned}
$$

We thus define a function

$$
\begin{aligned}
& \hat{\Phi}_{i j}\left(K-k, \bar{r}, \mu_{r}, M-m, \Omega-\omega\right)= \\
& \quad \int_{\mu_{x}=-\infty}^{\infty} \int_{\mu_{\phi}=-\pi}^{\pi} \int_{\tau=-\infty}^{\infty} R_{i j} \mathrm{e}^{\mathrm{i}(K-k) \mu_{x}} \mathrm{e}^{\mathrm{i}(M-m) \mu_{\phi}} \mathrm{e}^{-\mathrm{i}(\Omega-\omega) \tau} \mathrm{d} \tau \mathrm{d} \mu_{\phi} \mathrm{d} \mu_{x} ;
\end{aligned}
$$

this function is related to the turbulence velocity cross-spectrum $\Phi_{i j}$ by

$$
\hat{\Phi}_{i j}\left(K-k, \bar{r}, \Gamma_{M}-\gamma_{m}, M-m, \Omega-\omega\right)=\int_{\mu_{r}=-\infty}^{\infty} \hat{\Phi}_{i j} \mathrm{e}^{\mathrm{i}\left(\Gamma_{M}-\gamma_{m}\right) \mu_{r}}
$$

A function $\hat{S}$ for the turbulent entropy fluctuations is defined in the same way as $\hat{\Phi}$ for the velocity fluctuations; $S$ is the turbulent entropy cross-spectrum.

\subsection{The power spectral density of the scattered field}

The far-field power spectral density $\widetilde{P}_{M}(K, r, \Omega)$ is defined in terms of the transformed pressure field $\widetilde{P}_{M}^{\prime}(K, r, \Omega)$ as

$$
\tilde{P}_{M}(K, r, \Omega)=\left\langle\tilde{P}_{M}^{\prime} \tilde{P}_{M}^{\prime \star}\right\rangle
$$


For observers outside the cone of silence, equation (67) can be evaluated using the high-frequency solution (57), to give

$$
\begin{aligned}
& \tilde{P}_{M}(K, r, \Omega)=\left\langle\left(\frac{1}{(2 \pi)^{3}} \sum_{m_{1}=-\infty}^{\infty} \int_{\omega_{1}=-\infty}^{\infty} \int_{k_{1}=-\infty}^{\infty} \int_{r_{0_{1}}=0}^{\infty}-2 \mathrm{i} \frac{D_{0_{1}}^{2}}{d_{0_{1}}} r_{0_{1}} \tilde{G}_{M}\left(r \mid r_{0_{1}}\right) \tilde{p}_{m_{1}}^{\prime}\left(r_{0_{1}}\right) \Lambda\left(r_{0_{1}}\right) \times\right.\right. \\
& {\left[\frac{K}{D_{0_{1}}} \tilde{u}_{x t_{M-m_{1}}}^{\prime}\left(K-k_{1}, r_{0_{1}}, \Omega-\omega_{1}\right)+\frac{\Gamma_{M_{0_{1}}}}{D_{0_{1}}} \tilde{u}_{r t_{M-m_{1}}}^{\prime}\left(K-k_{1}, r_{0_{1}}, \Omega-\omega_{1}\right)\right.} \\
& \left.\left.+\frac{M / r_{0_{1}}}{D_{0_{1}}} \tilde{u}_{\phi t_{M-m_{1}}}^{\prime}\left(K-k_{1}, r_{0_{1}}, \Omega-\omega_{1}\right)+\frac{1}{2 C_{p}} \tilde{s}_{t_{M-m_{1}}}^{\prime}\left(K-k_{1}, r_{0_{1}}, \Omega-\omega_{1}\right)\right] \mathrm{d} r_{0_{1}} \mathrm{~d} k_{1} \mathrm{~d} \omega_{1}\right) \times \\
& \left(\frac{1}{(2 \pi)^{3}} \sum_{m_{2}=-\infty}^{\infty} \int_{\omega_{2}=-\infty}^{\infty} \int_{k_{2}=-\infty}^{\infty} \int_{r_{0_{2}}=0}^{\infty}+2 \mathrm{i} \frac{D_{0_{2}}^{2}}{d_{0_{2}}} r_{0_{2}} \tilde{G}_{M}^{\star}\left(r \mid r_{0_{2}}\right) \tilde{p}_{m_{2}}^{\prime \star}\left(r_{0_{2}}\right) \Lambda^{\star}\left(r_{0_{2}}\right) \times\right. \\
& {\left[\frac{K}{D_{0_{2}}} \tilde{u}_{x t_{M-m_{2}}}^{\prime \star \star}\left(K-k_{2}, r_{0_{2}}, \Omega-\omega_{2}\right)+\frac{\Gamma_{M_{0_{2}}}^{\star}}{D_{0_{2}}} \tilde{u}_{r t_{M-m_{2}}}^{\prime \star}\left(K-k_{2}, r_{0_{2}}, \Omega-\omega_{2}\right)\right.} \\
& \left.\left.\left.+\frac{M / r_{0_{2}}}{D_{0_{2}}} \tilde{u}_{\phi \phi_{M-m_{2}}^{\prime \star}}^{\prime \star}\left(K-k_{2}, r_{0_{2}}, \Omega-\omega_{2}\right)+\frac{1}{2 C_{p}} \tilde{s}_{t_{M-m_{2}}^{\prime *}}^{\prime \star}\left(K-k_{2}, r_{0_{2}}, \Omega-\omega_{2}\right)\right] \mathrm{d} r_{0_{2}} \mathrm{~d} k_{2} \mathrm{~d} \omega_{2}\right)\right\rangle \text {, }
\end{aligned}
$$

where $k_{1}, m_{1}, \omega_{1}, k_{2}, m_{2}, \omega_{2}, r_{01}$ and $r_{02}$ are all dummy variables.

On assuming the interchangeability of ensemble averaging and integration in equation (68), and applying the equation (64), we find

$$
\begin{aligned}
\tilde{P}_{M}(K, r, \Omega)= & \frac{1}{(2 \pi)^{3}} \sum_{m=-\infty}^{\infty} \int_{\omega=-\infty}^{\infty} \int_{k=-\infty}^{\infty} \int_{r_{0_{1}}=0}^{\infty} \int_{r_{0_{2}}=0}^{\infty} 2 r_{0_{1}} r_{0_{2}} \frac{D_{0_{1}}^{2} D_{0_{2}}^{2}}{d_{0_{1}} d_{0_{2}}} \times \\
& \tilde{G}_{M}\left(r \mid r_{0_{1}}\right) \tilde{G}_{M}^{\star}\left(r \mid r_{0_{2}}\right) \tilde{p}_{m}^{\prime}\left(r_{0_{1}}\right) \tilde{p}_{m}^{\prime \star}\left(r_{0_{2}}\right) \Lambda\left(r_{0_{1}}\right) \Lambda^{\star}\left(r_{0_{2}}\right) \times \\
& \left(\frac{4 K^{2}}{D_{0_{1}} D_{0_{2}}}\right) \hat{\Phi}_{x x}+\left(\frac{4 K \Gamma_{M_{0_{2}}}^{\star}}{D_{0_{1}} D_{0_{2}}}\right) \hat{\Phi}_{x r}+\left(\frac{4 K\left(M / r_{0_{2}}\right)}{D_{0_{1}} D_{0_{2}}}\right) \hat{\Phi}_{x \phi} \\
& +\left(\frac{4 \Gamma_{M_{0_{1}}} K}{D_{0_{1}} D_{0_{2}}}\right) \hat{\Phi}_{r x}+\left(\frac{4 \Gamma_{M_{0_{1}}} \Gamma_{M_{0_{2}}}^{\star}}{D_{0_{1}} D_{0_{2}}}\right) \hat{\Phi}_{r r}+\left(\frac{4 \Gamma_{M_{0_{1}}}\left(M / r_{0_{2}}\right)}{D_{0_{1}} D_{0_{2}}}\right) \hat{\Phi}_{r \phi} \\
& +\left(\frac{4\left(M / r_{0_{1}}\right) K}{D_{0_{1}} D_{0_{2}}}\right) \hat{\Phi}_{\phi x}+\left(\frac{4\left(M / r_{0_{1}}\right) \Gamma_{M_{0_{2}}}^{\star}}{D_{0_{1}} D_{0_{2}}}\right) \hat{\Phi}_{\phi r}+\left(\frac{4\left(M / r_{0_{1}}\right.}{D_{0_{1}} D_{0_{2}}}\right)\left(M / r_{0_{2}}\right) \\
& \left.+\frac{1}{C_{p}^{2}} \hat{S}\right] \mathrm{d} \omega \mathrm{d} k \mathrm{~d} r_{0_{1}} \mathrm{~d} r_{0_{2}} .
\end{aligned}
$$


From equation (31), the pressure terms are

$$
\begin{aligned}
\tilde{p}_{m}^{\prime}\left(r_{0_{1}}\right) \tilde{p}_{m}^{\prime \star}\left(r_{0_{2}}\right)= & \left|\tilde{p}_{m_{\infty}}^{\prime}\right|^{2}\left(\frac{1}{\sqrt{r_{0_{1}}} \sqrt{r_{0_{2}}}}\right)\left(\frac{\bar{\rho}_{0_{1}} \bar{\rho}_{0_{2}}}{\bar{\rho}_{\infty}^{2}}\right)^{1 / 2}\left(\frac{d_{0_{1}} d_{0_{2}}}{d_{\infty}^{2}}\right) \gamma_{m_{\infty}}\left(\frac{1}{\gamma_{m_{0_{1}}}}\right)^{1 / 2}\left(\frac{1}{\gamma_{m_{0_{2}}}^{\star}}\right)^{1 / 2} \times \\
& \exp \left\{\mathrm{i} \int_{r_{0_{1}}}^{r_{0_{2}}} \gamma_{m}\left(r^{\prime}\right) \mathrm{d} r^{\prime}\right\},
\end{aligned}
$$

and from equation (39), the Green's functions terms are

$$
\begin{aligned}
\tilde{G}_{M}\left(r \mid r_{0_{1}}\right) \tilde{G}_{M}^{\star}\left(r \mid r_{0_{2}}\right)= & \left(\frac{1}{4 r}\right)\left(\frac{1}{\sqrt{r_{0_{1}}} \sqrt{r_{0_{2}}}}\right)\left(\frac{\bar{\rho}_{\infty}^{2}}{\bar{\rho}_{0_{1}} \bar{\rho}_{0_{2}}}\right)^{1 / 2}\left(\frac{D_{\infty}^{2}}{D_{0_{1}}^{2} D_{0_{2}}^{2}}\right)\left(\frac{1}{\Gamma_{M_{\infty}}}\right) \times \\
& \left(\frac{1}{\Gamma_{M_{0_{1}}}}\right)^{1 / 2}\left(\frac{1}{\Gamma_{M_{0_{2}}}^{\star}}\right)^{1 / 2} \exp \left\{-\mathrm{i} \int_{r_{0_{1}}}^{r_{0_{2}}} \Gamma_{M}\left(r^{\prime}\right) \mathrm{d} r^{\prime}\right\} .
\end{aligned}
$$

Thus the far-field power spectral density is

$$
\begin{aligned}
& \tilde{P}_{M}(K, r, \Omega)=\frac{1}{(2 \pi)^{3}} \sum_{m=-\infty}^{\infty} \int_{\omega=-\infty}^{\infty} \int_{k=-\infty}^{\infty} \int_{r_{0_{1}}=0}^{\infty} \int_{r_{0_{2}}=0}^{\infty}\left|\tilde{p}_{m_{\infty}}^{\prime}\right|^{2} \frac{1}{2 r}\left(\frac{D_{\infty}}{d_{\infty}}\right)^{2}\left(\frac{\gamma_{m_{\infty}}}{\Gamma_{M_{\infty}}}\right) \times \\
& \left(\frac{1}{\gamma_{m_{0_{1}}}}\right)^{1 / 2}\left(\frac{1}{\gamma_{m_{0_{2}}}^{\star}}\right)^{1 / 2}\left(\frac{1}{\Gamma_{M_{0_{1}}}}\right)^{1 / 2}\left(\frac{1}{\Gamma_{M_{0_{2}}}^{\star}}\right)^{1 / 2} \Lambda\left(r_{0_{1}}\right) \Lambda^{\star}\left(r_{0_{2}}\right) \times \\
& {\left[\left(\frac{4 K^{2}}{D_{0_{1}} D_{0_{2}}}\right) \hat{\Phi}_{x x}+\left(\frac{4 K \Gamma_{M_{0_{2}}}^{\star}}{D_{0_{1}} D_{0_{2}}}\right) \hat{\Phi}_{x r}+\left(\frac{4 K\left(M / r_{0_{2}}\right)}{D_{0_{1}} D_{0_{2}}}\right) \hat{\Phi}_{x \phi}\right.} \\
& +\left(\frac{4 \Gamma_{M_{0_{1}}} K}{D_{0_{1}} D_{0_{2}}}\right) \hat{\Phi}_{r x}+\left(\frac{4 \Gamma_{M_{0_{1}}} \Gamma_{M_{0_{2}}}^{\star}}{D_{0_{1}} D_{0_{2}}}\right) \hat{\Phi}_{r r}+\left(\frac{4 \Gamma_{M_{0_{1}}}\left(M / r_{0_{2}}\right)}{D_{0_{1}} D_{0_{2}}}\right) \hat{\Phi}_{r \phi} \\
& +\left(\frac{4\left(M / r_{0_{1}}\right) K}{D_{0_{1}} D_{0_{2}}}\right) \hat{\Phi}_{\phi x}+\left(\frac{4\left(M / r_{0_{1}}\right) \Gamma_{M_{0_{2}}}^{\star}}{D_{0_{1}} D_{0_{2}}}\right) \hat{\Phi}_{\phi r}+\left(\frac{4\left(M / r_{0_{1}}\right)\left(M / r_{0_{2}}\right)}{D_{0_{1}} D_{0_{2}}}\right) \hat{\Phi}_{\phi \phi} \\
& \left.+\frac{1}{C_{p}^{2}} \hat{S}\right] \exp \left\{-\mathrm{i} \int_{r_{0_{1}}}^{r_{0_{2}}}\left(\Gamma_{M}\left(r^{\prime}\right)-\gamma_{m}\left(r^{\prime}\right)\right) d r^{\prime}\right\} \mathrm{d} \omega \mathrm{d} k \mathrm{~d} r_{0_{1}} \mathrm{~d} r_{0_{2}} \text {. }
\end{aligned}
$$

To further simplify equation (72), we make an assumption that the correlation length of the turbulence is small. To this end, we change the variables in equation (72) from 
radii $r_{0_{1}}, r_{0_{2}}$ to the seperation $\mu_{r}$ and mean position $\bar{r}$ given by equations (62) and (63). Under this change of variable, the double integral in equation (72) transforms as

$$
\int_{r_{0_{1}}=0}^{\infty} \int_{r_{0_{2}}=0}^{\infty} \mathrm{d} r_{01} \mathrm{~d} r_{02} \rightarrow \int_{\bar{r}=0}^{\infty} \int_{\mu_{r}=-\infty}^{\infty} \mathrm{d} \bar{r} \mathrm{~d} \mu_{r}
$$

It is assumed that the radial turbulence correlations in (72) are negligible unless $\mu_{r}$ is sufficiently small. Let the evaluation of a function at $\bar{r}$ be denoted with an overbar on the function, so for example $D(\bar{r})=\bar{D}$. Then for a general function $f(r)$,

$$
f\left(r_{01}\right) f\left(r_{02}\right)=[f(\bar{r})]^{2}+\mathrm{O}\left(\mu_{r}^{2}\right)
$$

and we make the approximations

$$
\gamma_{m}\left(r_{01}\right) \approx \bar{\gamma}_{m}, \quad \Gamma_{m}\left(r_{01}\right) \approx \bar{\Gamma}_{m}, \quad D\left(r_{01}\right) \approx \bar{D}, \quad \Lambda_{m}\left(r_{01}\right) \approx \bar{\Lambda}_{m}
$$

and

$$
\gamma_{m}\left(r_{02}\right) \approx \bar{\gamma}_{m}, \quad \Gamma_{m}\left(r_{02}\right) \approx \bar{\Gamma}_{m}, \quad D\left(r_{02}\right) \approx \bar{D}, \quad \Lambda_{m}\left(r_{02}\right) \approx \bar{\Lambda}_{m} .
$$

In the exponent of equation (72), we make the approximation

$$
\int_{r_{0_{1}}}^{r_{0_{2}}}\left(\Gamma_{M}\left(r^{\prime}\right)-\gamma_{m}\left(r^{\prime}\right)\right) \mathrm{d} r^{\prime} \approx-\mu_{r}\left(\bar{\Gamma}_{M}-\bar{\gamma}_{m}\right)
$$

Under these approximations, equation (72) becomes

$$
\begin{aligned}
\tilde{P}_{M}(K, r, \Omega) \approx & \frac{1}{(2 \pi)^{3}} \sum_{m=-\infty}^{\infty} \int_{\omega=-\infty}^{\infty} \int_{k=-\infty}^{\infty} \int_{\bar{r}=0}^{\infty} \int_{\mu_{r}=-\infty}^{\infty}\left|\tilde{p}_{m_{\infty}}^{\prime}\right|^{2} \frac{1}{2 r}\left(\frac{D_{\infty}}{d_{\infty}}\right)^{2}\left(\frac{\gamma_{m_{\infty}}}{\Gamma_{M_{\infty}}}\right) \times \\
& \left(\frac{1}{\bar{\gamma}_{m}^{2}}\right)^{1 / 2}\left(\frac{1}{\bar{\Gamma}_{M}^{2}}\right)^{1 / 2}\left(K k+\bar{\Gamma}_{M} \bar{\gamma}_{m}+\frac{M m}{\bar{r}^{2}}\right)^{2} \frac{4}{\bar{D}^{2}} \times \\
& {\left[K^{2} \hat{\Phi}_{x x}+\bar{\Gamma}_{M}^{2} \hat{\Phi}_{r r}+\frac{M^{2}}{\bar{r}^{2}} \hat{\Phi}_{\phi \phi}+K \bar{\Gamma}_{M} \hat{\Phi}_{r r}+\bar{\Gamma}_{M} K \hat{\Phi}_{r x}+K \frac{M}{\bar{r}} \hat{\Phi}_{r \phi}\right.} \\
& \left.+\frac{M}{\bar{r}} K \hat{\Phi}_{\phi x}+\bar{\Gamma}_{M} \frac{M}{\bar{r}} \hat{\Phi}_{r \phi}+\frac{M}{\bar{r}} \bar{\Gamma}_{M} \hat{\Phi}_{\phi r}+\frac{\bar{D}^{2}}{4 C_{p}^{2}} \hat{S}\right] \\
& \exp \left\{\mathrm{i} \mu_{r}\left(\bar{\Gamma}_{M}-\bar{\gamma}_{m}\right)\right\} \mathrm{d} \omega \mathrm{d} k \mathrm{~d} \bar{r} \mathrm{~d} \mu_{r} .
\end{aligned}
$$


The integral over $\mu_{r}$ in this expression is equivalent to a Fourier transform, and from equation (66) can be evaluated to give

$$
\begin{aligned}
\tilde{P}_{M}(K, r, \Omega) \approx & \frac{1}{(2 \pi)^{3}} \sum_{m=-\infty}^{\infty} \int_{\omega=-\infty}^{\infty} \int_{k=-\infty}^{\infty} \int_{\bar{r}=0}^{\infty}\left|\tilde{p}_{m_{\infty}^{\prime}}\right|^{2} \frac{1}{2 r}\left(\frac{D_{\infty}}{d_{\infty}}\right)^{2}\left(\frac{\gamma_{m_{\infty}}}{\Gamma_{M_{\infty}}}\right) \times \\
& \left(\frac{1}{\bar{\gamma}_{m}^{2}}\right)^{1 / 2}\left(\frac{1}{\bar{\Gamma}_{M}^{2}}\right)^{1 / 2}\left(K k+\bar{\Gamma}_{M} \bar{\gamma}_{m}+\frac{M m}{\bar{r}^{2}}\right)^{2} \frac{4}{\bar{D}^{2}} \times \\
& {\left[K^{2} \Phi_{x x}+\bar{\Gamma}_{M}^{2} \Phi_{r r}+\frac{M^{2}}{\bar{r}^{2}} \Phi_{\phi \phi}+K \bar{\Gamma}_{M} \Phi_{x r}+\bar{\Gamma}_{M} K \Phi_{r x}\right.} \\
& \left.+K \frac{M}{\bar{r}} \Phi_{x \phi}+\frac{M}{\bar{r}} K \Phi_{\phi x}+\bar{\Gamma}_{M} \frac{M}{\bar{r}} \Phi_{r \phi}+\frac{M}{\bar{r}} \bar{\Gamma}_{M} \Phi_{\phi r}+\frac{\bar{D}^{2}}{4 C_{p}^{2}} S\right] \mathrm{d} \omega \mathrm{d} k \mathrm{~d} \bar{r},
\end{aligned}
$$

where $\Phi_{i j}$ and $S$ are the turbulence velocity and entropy cross-spectra, evaluated at the difference wavenumbers and frequencies $\left(K-k, \bar{r}, \Gamma_{M}-\gamma_{m}, M-m, \Omega-\omega\right)$. This solution can be written in the form

$$
\tilde{P}_{M}(K, r, \Omega) \approx \frac{1}{r} \frac{1}{(2 \pi)^{3}} \sum_{m=-\infty}^{\infty} \int_{\omega=-\infty}^{\infty} \int_{k=-\infty}^{\infty}\left|\tilde{p}_{m_{\infty}}^{\prime}\right|^{2} \mathcal{H}(K, M, \Omega, k, m, \omega) \mathrm{d} \omega \mathrm{d} k
$$

where $\mathcal{H}(K, M, \Omega, k, m, \omega)$ is given by an integral over the source region:

$$
\begin{aligned}
\mathcal{H}(K, M, \Omega, k, m, \omega)= & \int_{\bar{r}=0}^{\infty}\left(\frac{D_{\infty}}{d_{\infty}}\right)^{2}\left(\frac{\gamma_{m_{\infty}}}{\Gamma_{M_{\infty}}}\right)\left(\frac{1}{\bar{\gamma}_{m}^{2}}\right)^{1 / 2}\left(\frac{1}{\bar{\Gamma}_{M}^{2}}\right)^{1 / 2}\left(K k+\bar{\Gamma}_{M} \bar{\gamma}_{m}+\frac{M m}{\bar{r}^{2}}\right)^{2} \frac{2}{\bar{D}^{2}} \times \\
& {\left[K^{2} \Phi_{x x}+\bar{\Gamma}_{M}^{2} \Phi_{r r}+\frac{M^{2}}{\bar{r}^{2}} \Phi_{\phi \phi}+K \bar{\Gamma}_{M} \Phi_{x r}+\bar{\Gamma}_{M} K \Phi_{r x}\right.} \\
& \left.+K \frac{M}{\bar{r}} \Phi_{x \phi}+\frac{M}{\bar{r}} K \Phi_{\phi x}+\bar{\Gamma}_{M} \frac{M}{\bar{r}} \Phi_{r \phi}+\frac{M}{\bar{r}} \bar{\Gamma}_{M} \Phi_{\phi r}+\frac{\bar{D}^{2}}{4 C_{p}^{2}} S\right] \mathrm{d} \bar{r}
\end{aligned}
$$

\subsection{Far-field directivity}

Equation (80) gives the far-field power spectrum of the scattered field, outside the cone of silence, in terms of the wavenumber $K$ and cylindrical radius $r$. In general however, we will be interested in evaluating the spectrum at a given polar angle $\Theta$ at a spherical distance $R$ from the source. To write the power spectrum in such a form, we first show how to transform the scattered pressure $\widetilde{P}^{\prime}{ }_{M}(K, r, \Omega)$ to a function $\widetilde{P}_{M}^{\prime}(R, \Theta, \Omega)$. 
In the far field, outside the shear layer, the turbulence intensity is zero, so the scattering equation is homogeneous: thus the scattered field must in the far field be of the form of the homogeneous solution (31). Thus we may write, as $r \rightarrow \infty$,

$$
\tilde{P}_{M}^{\prime}(K, r, \Omega) \sim \frac{\tilde{A}_{M_{\infty}}(K, \Omega)}{\sqrt{r}} \exp \left\{-\mathrm{i} \Gamma_{M_{\infty}} r\right\}
$$

where $\Gamma_{M_{\infty}}>0$. The inverse transform of equation (82) is defined as

$$
\tilde{P}_{M}^{\prime}(x, r, \Omega) \sim \frac{1}{2 \pi} \int_{K=-\infty}^{\infty} \frac{\tilde{A}_{M_{\infty}}(K, \Omega)}{\sqrt{r}} \mathrm{e}^{-\mathrm{i}\left(K x+\Gamma_{M_{\infty}} r\right)} \mathrm{d} K, \quad \text { as } r \rightarrow \infty .
$$

Note that since $U_{\infty}=0$, equation (37) leads to $\Gamma_{M_{\infty}}=K_{\infty}^{2}-K^{2}$, where $K_{\infty}=\Omega / \bar{c}_{\infty}$. Thus in practice the limits on the integral in equation (83) can be taken as $-\left|K_{\infty}\right|$ and $\left|K_{\infty}\right|$, since for other values of $K$ the field is evanescent and does not propagate to the far field. Conversion in equation (83) from cylindrical to spherical coordinates gives

$$
\tilde{P}_{M}^{\prime}(R, \Theta, \Omega) \sim \frac{1}{2 \pi} \frac{1}{\sqrt{R \sin \Theta}} \int_{K=-\left|K_{\infty}\right|}^{\left|K_{\infty}\right|} \tilde{A}_{M_{\infty}}(K, \Omega) \mathrm{e}^{-\mathrm{i} R\left(K \cos \Theta+\Gamma_{M_{\infty}} \sin \Theta\right)} \mathrm{d} K, \text { as } R \rightarrow \infty .
$$

The integral in this equation is of a form suitable for evaluation by the method of stationary phase, a high-frequency asymptotic method described by Bleistein and Handelsman [20]. The result is

$$
\tilde{P}_{M}^{\prime}(R, \Theta, \Omega) \sim \frac{1}{\sqrt{2 \pi}} \frac{\sqrt{\left|K_{\infty}\right| \sin \Theta}}{R} \tilde{A}_{M_{\infty}}\left(\left|K_{\infty}\right| \cos \Theta, \Omega\right) \mathrm{e}^{-\mathrm{i}\left|K_{\infty}\right| R} \mathrm{e}^{\mathrm{i} \pi / 4},
$$

where the stationary phase point is $K=\left|K_{\infty}\right| \cos \Theta$.

The far-field power spectral density is defined by

$$
\tilde{P}_{M}(K, r, \Omega)=\left\langle\tilde{P}_{M}^{\prime}(K, r, \Omega) \tilde{P}_{M}^{\prime \star}(K, r, \Omega)\right\rangle \text { as } r \rightarrow \infty,
$$

which, from equation (82) is equivalent to

$$
\tilde{P}_{M}(K, r, \Omega)=\frac{1}{r}\left\langle\tilde{A}_{M_{\infty}}(K, \Omega) \tilde{A}_{M_{\infty}}^{\star}(K, \Omega)\right\rangle .
$$


Define $\tilde{P}_{M_{D}}(\Theta, \Omega)$ as the far-field directivity of the mean-square pressure (azimuthal order $M$ ) of the scattered acoustic field; then

$$
\begin{aligned}
\tilde{P}_{M_{D}}(\Theta, \Omega) & =R^{2}\left\langle\tilde{P}_{M}^{\prime}(R, \Theta, \Omega) \tilde{P}_{M}^{\prime \star}(R, \Theta, \Omega)\right\rangle, \\
& \sim \frac{1}{2 \pi}\left|K_{\infty}\right| \sin \Theta\left\langle\tilde{A}_{M_{\infty}}\left(\left|K_{\infty}\right| \cos \Theta, \Omega\right) \tilde{A}_{M_{\infty}}^{\star}\left(\left|K_{\infty}\right| \cos \theta, \Omega\right)\right\rangle,
\end{aligned}
$$

as $R \rightarrow \infty$. Then comparison of equations (87) and (89) gives the relationship

$$
\tilde{P}_{M_{D}}(\Theta, \Omega) \sim \frac{1}{2 \pi}\left|K_{\infty}\right| \sin \Theta\left(r \tilde{P}_{M}\left(\left|K_{\infty}\right| \cos \Theta, r, \Omega\right)\right)
$$

between the scattered spectrum and directivity. An identical analysis for the incident field leads to

$$
\tilde{p}_{m_{d}}(\theta, \omega) \sim \frac{1}{2 \pi}\left|k_{\infty}\right| \sin \theta\left(r \tilde{p}_{m}\left(\left|k_{\infty}\right| \cos \theta, r, \omega\right)\right) .
$$

For the above results to be substituted into equation (80), the integral over $k$ must be transformed into an integral over $\theta$, by the change of variable $k=\left|k_{\infty}\right| \cos \theta$, with $0 \leq$ $\theta \leq \pi$. The integral transforms as

$$
\int_{k=-\infty}^{\infty} \mathrm{d} k \rightarrow \int_{k=-\left|k_{\infty}\right|}^{\left|k_{\infty}\right|} \mathrm{d} k \rightarrow \int_{\theta=0}^{\pi}\left|k_{\infty}\right| \sin \theta \mathrm{d} \theta
$$

With this change of variable, equations (90) and (91) can be substituted into equation (80), to give

$$
\begin{aligned}
\tilde{P}_{M_{D}}(\Theta, \Omega) \sim & \left|K_{\infty}\right| \sin \Theta \frac{1}{(2 \pi)^{3}} \sum_{m=-\infty}^{\infty} \int_{\omega=-\infty}^{\infty} \int_{\theta=0}^{\pi} \tilde{p}_{m_{d}}(\theta, \omega) \times \\
& \mathcal{H}\left(\left|K_{\infty}\right| \cos \Theta, M, \Omega,\left|k_{\infty}\right| \cos \theta, m, \omega\right) \mathrm{d} \omega \mathrm{d} \theta .
\end{aligned}
$$

\subsection{Summary of results}

Equation (93) expresses the far-field mean-square pressure directivity of the scattered field, $\tilde{P}_{M_{D}}(\Theta, \Omega)$, in terms of the far-field mean-square pressure directivity of the 
incident field, $\tilde{p}_{m_{d}}(\theta, \omega)$. The result has the incident field directivity multiplied by a function $\mathcal{H}$, specified by equation (81), which is an integral over the turbulent shear layer of a function dependent on the mean-flow properties and the turbulence crossspecta. The result is integrated over the incident frequency $\omega$ and over the range of incident angles $\theta$, then summed over all modes of the incident field. The theory on which equation (93) is based is valid for observers in the far field, outside the cone of silence of the jet, in the case of weak scattering.

\section{COMPARISON WITH EXPERIMENT 3.1. The Candel experiment}

There are few published examples of experimental data on tone haystacking, and of the results available, that most suitable for validation of the current model is the work of Candel et al [1]-[5]. In reference [1], the authors describe an experiment conducted at the von Karman Institute in the $3 \mathrm{~m}$ diameter open jet wind tunnel. A sketch of the expermental setup is shown in figure 4. A monochromatic source was placed at the centreline of the jet, and the radiated sound passed through the turbulent shear layer to reach the microphone placed outside the jet. The microphone was positioned at a polar angle of $90^{\circ}$ from the source. Measurements were taken with this configuration of source and microphone at two positions along the jet, the first at a distance of $0.5 \mathrm{~m}$ from the nozzle, the second at a distance $1.95 \mathrm{~m}$ from the nozzle. The haystacked field was measured for a range of source frequencies from $4 \mathrm{kHz}$ to $20 \mathrm{kHz}$, and the jet velocity was varied from $20 \mathrm{~ms}^{-1}$ to $60 \mathrm{~ms}^{-1}$.

Further, Candel et al [1] provide estimates of the mean-flow velocity profile $U(r)$, the turbulence convection velocity $U_{c}$, and the turbulence lengthscale; measurements of the

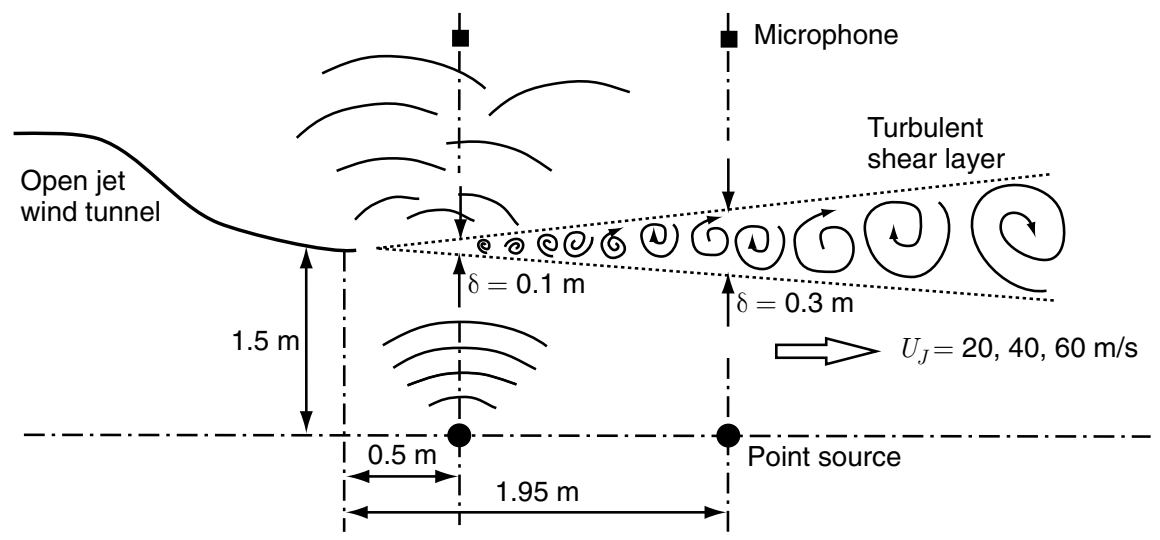

Figure 4: A point source is placed on the centreline of a jet, and a microphone placed outside the jet at a polar angle approximately $90^{\circ}$ from the source. 
the axial and radial turbulence intensity are also given (peak levels approximately 15\%). For the mean-flow velocity, we take

$$
U(r)=U_{J} \frac{1}{2}(1-\tanh \eta), \quad \eta=\frac{2}{\delta}\left(r-r_{c}\right)
$$

where $U_{J}$ is the jet centreline velocity, $\delta$ is the shear layer vorticity thickness, and $r_{c}$ is the jet radius. In reference [5], Candel et al estimate the shear layer thickness and jet radius as

$$
\delta=0.17 x, \quad r_{c}=1.47+0.048 x
$$

where $x$ is the distance in metres downstream of the nozzle; the convection velocity of the turbulent eddies is estimated as $U_{c} \approx 0.5 U_{J}$, and the turbulence correlation lengthscale is estimated to be $l \approx 3.25 \delta$, though this seems very large. In addition, the measured far-field radiation pattern is shown to be fairly omnidirectional, so in our simulations we assume the incident-field directivity is uniform outside the cone of silence. Since the jet is isothermal, $\bar{c}$ and $\bar{\rho}$ are assumed to be uniform.

To model Candel et al's experiment with our analytical solution (93), we must apply the cross-spectra of the turbulent velocity and entropy fluctuations. Since the jet is unheated, we assume that the turbulent entropy fluctuations are negligible, and that the velocity fluctuations are the primary scattering mechanism. We shall present two possible models for the turbulent velocity spectrum $\Phi_{i j}$; the first is a standard Gaussian correlation, and the second we refer to as a Gaussian-HIT correlation, since theory related to Homogeneous Isotropic Turbulence is used to derive this function. In both instances, we shall apply a frozen turbulence model, assuming that the turbulence convects downstream at velocity $U_{c}$, but is otherwise time-independent. This should be a reasonable approximation so long as the real turbulence correlation timescale is sufficiently large. Candel's measurements show that the turbulence is not truly isotropic, but the difference in radial and axial intensities is small. We model the turbulence as isotropic (using Candel's $\Phi_{r r}$ to determine the intensity) and axially homogeneous, and confined within the shear layer.

\subsection{Study 1: Gaussian correlation}

In the first instance we take a standard Gaussian turbulent velocity cross-correlation function. Then, in terms of the separation variables (62), the correlation function is

$$
R_{i j}=\overline{u_{t i}^{\prime} u_{t j}^{\prime}}(\bar{r}) \exp \left\{-\left(\frac{\left(\mu_{x}-U_{c} \tau\right)^{2}+\mu_{r}^{2}+\bar{r}^{2} \mu_{\phi}^{2}}{l^{2}}\right)\right\}
$$


Now we assume that $\overline{u_{t i}^{\prime} u_{t j}^{\prime}}=0$ for $i \neq j$; measured values for $i=j$ are given by Candel et al [1]. The cross-spectra are then given by a Fourier transform as

$$
\begin{gathered}
\Phi_{x x}=\frac{\overline{u_{t x}^{\prime} u_{t x}^{\prime}}(\bar{r})}{\bar{r}} l^{3} \pi^{\frac{3}{2}} \Pi \exp \left\{-\frac{l^{2}}{4}\left((K-k)^{2}+(\Gamma-\gamma)^{2}+\frac{(M-m)^{2}}{\bar{r}^{2}}\right)\right\}, \\
\Phi_{r r}=\frac{\overline{u_{t r}^{\prime} u_{t r}^{\prime}}(\bar{r})}{\bar{r}} l^{3} \pi^{\frac{3}{2}} \Pi \exp \left\{-\frac{l^{2}}{4}\left((K-k)^{2}+(\Gamma-\gamma)^{2}+\frac{(M-m)^{2}}{\bar{r}^{2}}\right)\right\}, \\
\Phi_{\phi \phi}=\frac{\overline{u_{t \phi}^{\prime} u_{t \phi}^{\prime}}(\bar{r})}{\bar{r}} l^{3} \pi^{\frac{3}{2}} \Pi \exp \left\{-\frac{l^{2}}{4}\left((K-k)^{2}+(\Gamma-\gamma)^{2}+\frac{(M-m)^{2}}{\bar{r}^{2}}\right)\right\},
\end{gathered}
$$

where the function II arises from the frozen turbulence assumption, and is given by

$$
\Pi=2 \pi \delta\left((\Omega-\omega)-(K-k) U_{c}\right)
$$

Given the spectra (97)-(99), the solution (93) has been evaluated for the cases considered in Candel et al [1]. We use the measured values from the experiment for most of the required parameters, but given the very large correlation length reported by Candel, we instead assume a more reasonable value of $l=\delta$ (see discussion below, section 3.4). Results are shown in figure 5, for a sum of scattered azimuthal orders $M=-100$ to $M=100$. Note that the absolute level of the source used in the experiment is not known; in both the experiment and the model analysis the broadband levels are plotted relative to the incident tone level, which is normalised to $0 \mathrm{~dB}$.

In general for these results, the 'double-humped' shape of the noise spectra are not reproduced, the absolute levels of the broadband do not match the experimental measurements, and the spectra are too narrow. However, the relative levels between predictions, say for different frequencies, are correctly predicted. We will now show how an alternative choice of correlation function improves these results.

\subsection{Study 2: Gaussian-HIT correlation}

Though the Gaussian correlation function is commonly used to describe turbulent flows, we here consider an alternative formulation. Batchelor [21] has shown that in homogeneous isotropic turbulence, the longitudinal and lateral velocity correlations are related to one another in a certain manner. This concept has been discussed more 
(a)

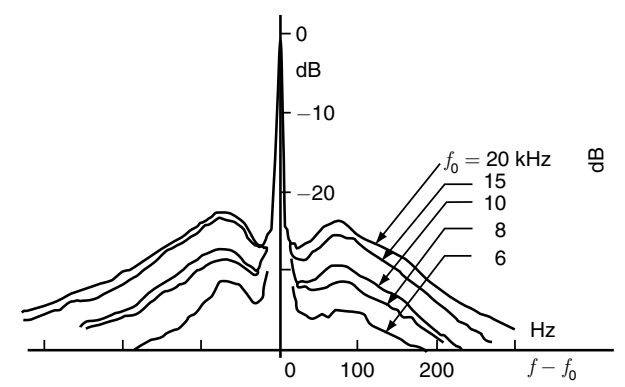

(b)

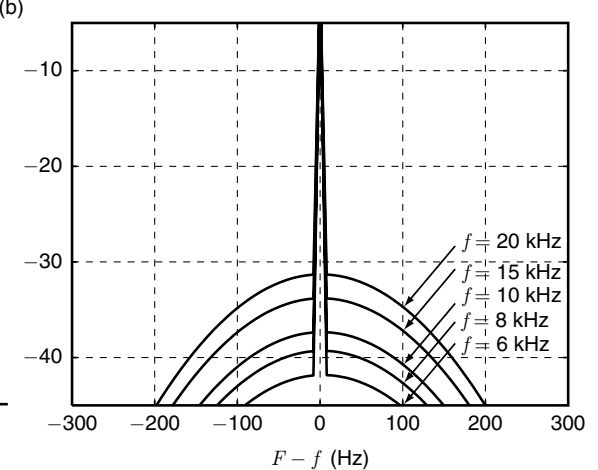

(c)

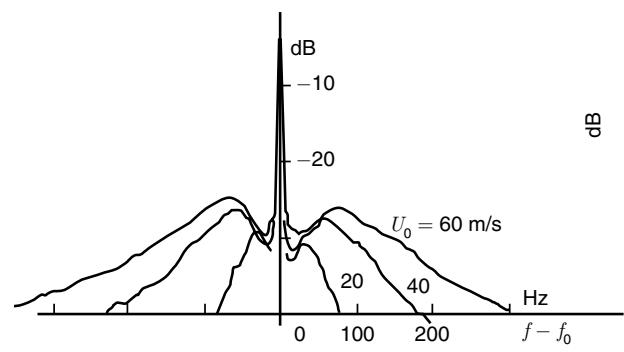

(d)

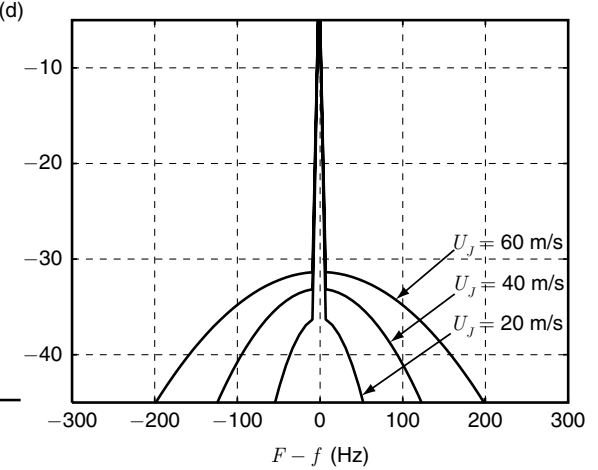

(e)

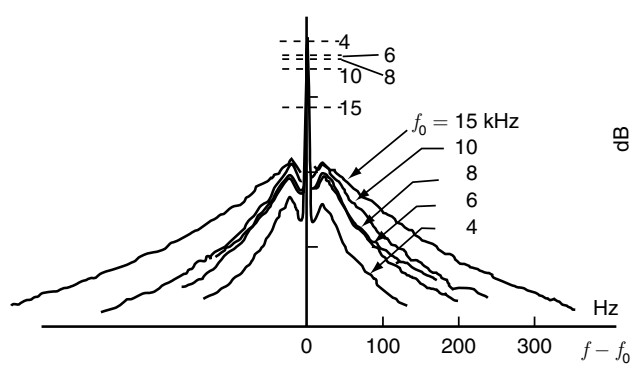

$(\mathrm{f})$

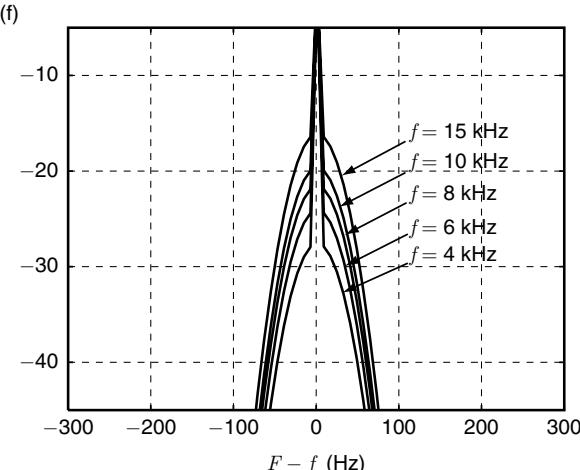

Figure 5: Scattered acoustic field at $\Theta=90^{\circ}$. Measurements and predictions of power spectra normalized by the total power. Prediction uses Gaussian turbulence correlation function, (a-b) Effect of varying source frequency at source position $x=0.5 \mathrm{~m}$, with $U_{J}=60 \mathrm{~m} / \mathrm{s}$. (c-d) Effect of varying jet velocity at source position $x=0.5 \mathrm{~m}$, with $f_{0}=20 \mathrm{kHz}$. (e-f) Effect of varying source frequency at source position $x=1.95 \mathrm{~m}$, with $U_{J}=60 \mathrm{~m} / \mathrm{s}$. Figures a,c,e reproduced from Candel et al [1], (1975 IEEE. 
recently by Ewert [22]. Through consideration of this relationship, a Gaussian-HIT (Homogeneous Isotropic Turbulence) correlation function can be derived. This correlation function is

$$
\begin{gathered}
R_{x x}=\overline{u_{t x}^{\prime} u_{t x}^{\prime}}(\bar{r})\left(1-\frac{\mu_{r}^{2}}{l^{2}}\right)\left(1-\frac{\bar{r}^{2} \mu_{\phi}^{2}}{l^{2}}\right) \exp \left\{-\left(\frac{\left(\mu_{x}-U_{c} \tau\right)^{2}+\mu_{r}^{2}+\bar{r}^{2} \mu_{\phi}^{2}}{l^{2}}\right)\right\}, \\
R_{r r}=\overline{u_{t r}^{\prime} u_{t r}^{\prime}}(\bar{r})\left(1-\frac{\left(\mu_{x}-U_{c} \tau\right)^{2}}{l^{2}}\right)\left(1-\frac{\bar{r}^{2} \mu_{\phi}^{2}}{l^{2}}\right) \exp \left\{-\left(\frac{\left(\mu_{x}-U_{c} \tau\right)^{2}+\mu_{r}^{2}+\bar{r}^{2} \mu_{\phi}^{2}}{l^{2}}\right)\right\}, \\
R_{\phi \phi}=\overline{u_{t \phi}^{\prime} u_{t \phi}^{\prime}}(\bar{r})\left(1-\frac{\left(\mu_{x}-U_{c} \tau\right)^{2}}{l^{2}}\right)\left(1-\frac{\mu_{r}^{2}}{l^{2}}\right) \exp \left\{-\left(\frac{\left(\mu_{x}-U_{c} \tau\right)^{2}+\mu_{r}^{2}+\bar{r}^{2} \mu_{\phi}^{2}}{l^{2}}\right)\right\}
\end{gathered}
$$

The corresponding spectra are

$$
\begin{aligned}
& \Phi_{x x}=\frac{\overline{u_{t x}^{\prime} u_{t x}^{\prime}}(\bar{r})}{4 \bar{r}} l^{3} \pi^{\frac{3}{2}} \Pi\left(1+\frac{l^{2}(\Gamma-\gamma)^{2}}{2}\right)\left(1+\frac{l^{2}(M-m)^{2}}{2 \bar{r}^{2}}\right) \times \\
& \exp \left\{-\frac{l^{2}}{4}\left((K-k)^{2}+(\Gamma-\gamma)^{2}+\frac{(M-m)^{2}}{\bar{r}^{2}}\right)\right\} \text {, } \\
& \Phi_{r r}=\frac{\overline{u_{t r}^{\prime} u_{t r}^{\prime}}(\bar{r})}{4 \bar{r}} l^{3} \pi^{\frac{3}{2}} \Pi\left(1+\frac{l^{2}(K-k)^{2}}{2}\right)\left(1+\frac{l^{2}(M-m)^{2}}{2 \bar{r}^{2}}\right) \times \\
& \exp \left\{-\frac{l^{2}}{4}\left((K-k)^{2}+(\Gamma-\gamma)^{2}+\frac{(M-m)^{2}}{\bar{r}^{2}}\right)\right\} \text {, } \\
& \Phi_{\phi \phi}=\frac{\overline{u_{t \phi}^{\prime} u_{t \phi}^{\prime}}(\bar{r})}{4 \bar{r}} l^{3} \pi^{\frac{3}{2}} \Pi\left(1+\frac{l^{2}(K-k)^{2}}{2}\right)\left(1+\frac{l^{2}(\Gamma-\gamma)^{2}}{2}\right) \times \\
& \exp \left\{-\frac{l^{2}}{4}\left((K-k)^{2}+(\Gamma-\gamma)^{2}+\frac{(M-m)^{2}}{\bar{r}^{2}}\right)\right\}
\end{aligned}
$$

where, as before, the frozen turbulence assumption gives the term II specified in equation (100).

Given the spectra (104)-(106), the solution (93) has again been evaluated for the cases considered in Candel et al [1]. Results are shown in figure 6, for a sum of scattered azimuthal orders $M=-100$ to $M=100$. 
(a)

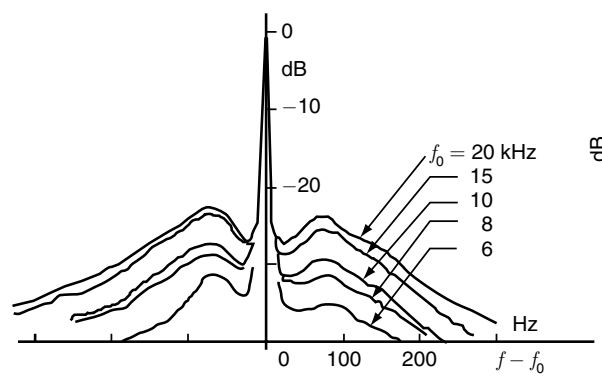

(b)

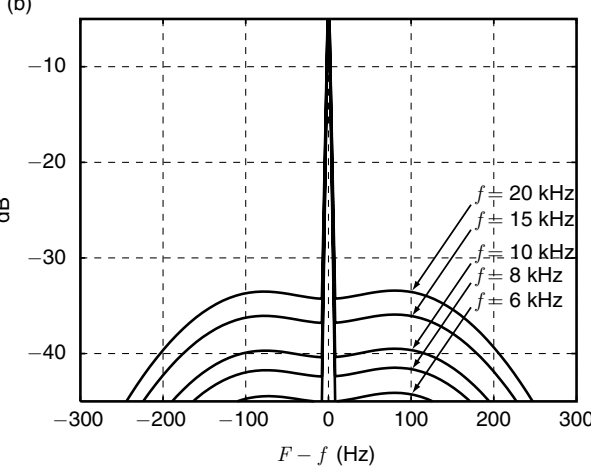

(c)

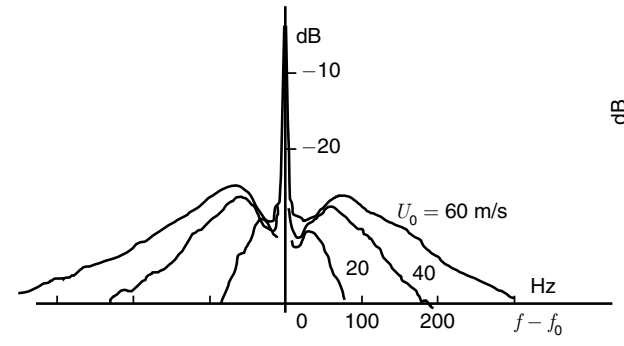

(d)

m

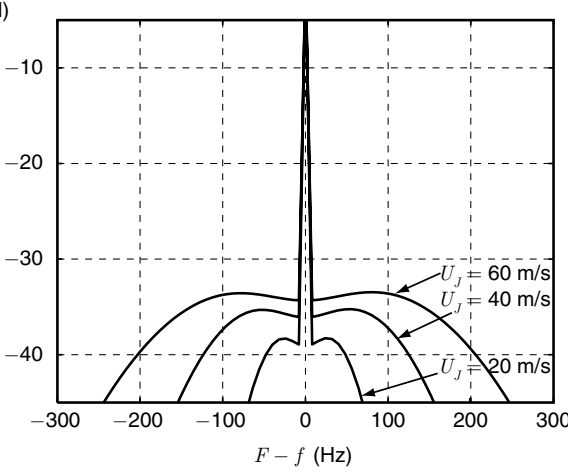

(f)

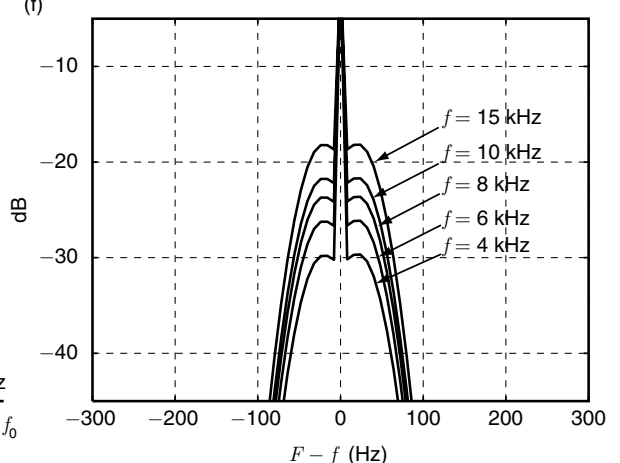

Figure 6: Scattered acoustic field at $\Theta=90^{\circ}$. Measurements and predictions of power spectra normalized by the total power. Prediction uses GaussianHIT turbulence correlation function, (a-b) Effect of varying source frequency at source position $x=0.5 \mathrm{~m}$, with $U_{J}=60 \mathrm{~m} / \mathrm{s}$. (c-d) Effect of varying jet velocity at source position $x=0.5 \mathrm{~m}$, with $f_{0}=20 \mathrm{kHz}$. (e-f) Effect of varying source frequency at source position $x=1.95 \mathrm{~m}$, with $U_{J}=60 \mathrm{~m} / \mathrm{s}$. Figures a,c,e reproduced from Candel et al [1], (C)1975 IEEE. 
In these results, the 'double-humped' shape of the spectra are reproduced: this is due to the fact that the turbulent velocity cross-spectra (104)-(106) are themselves of a 'double-humped' form. The predicted noise spectra are now less narrow than in the case of Gaussian turbulence, and this corresponds more closely to the experimental data. At large separation frequencies $F-f$, the predicted broadband levels fall away more quickly than in the experimental data: this may be due to the frozen turbulence assumption. In the predicted spectra, the absolute levels of the predictions are too low compared to the experimental measurements. This may be partially due to the approximation made at equation (40): recall that we essentially ignored the reflected ray in making the approximation $X=1$. Summing the direct and the reflected rays incoherently would raise the predicted levels by $3 \mathrm{~dB}$. Note however that the relative levels between predictions, say for different frequencies in part (a) of figure 6 , are correctly predicted. In varying frequency or velocity, the model correctly predicts the trends in the behaviour of the scattered field. Note that both the Gaussian and the Gaussian-HIT models give similar predictions for the relative levels of the scattering for different frequencies and velocities: the Gaussian HIT model is an improvement over the Gaussian model only in that the shape of the spectrum is more accurately predicted.

\subsection{Spectral shape at $\Theta=90^{\circ}$}

In the experimental results of Candel, it is noted that the power spectral density of the scattered field is of a 'double-humped' form, with a local maximum at some distance either side of the tone. This behaviour is also observed in the predictions made using the Gaussian HIT turbulence model (see figure 6). The location of these local maxima can be easily predicted using the turbulence spectra and the frozen turbulence assumption.

The Gaussian HIT turbulence spectra (104-106) contain terms of the form

$$
\left(1+\frac{l^{2}(K-k)^{2}}{2}\right) \exp \left(-\frac{(K-k)^{2} l^{2}}{4}\right),
$$

which have local maxima at wavenumbers $(K-k) l= \pm \sqrt{2}$. The frozen turbulence assumption gives the delta function in the turbulence spectra, relating frequencies and wavenumbers by

$$
(\Omega-\omega)-U_{c}(K-k)=0 .
$$

The tone-to-peak seperation in the spectrum of the scattered field can then be predicted as

$$
\Delta f= \pm \frac{\sqrt{2}}{2 \pi} \frac{U_{c}}{l} .
$$


This result directly relates the shape of the haystack to the basic properties of the turbulence, and can be compared to the result of Candel [1], who assumed that

$$
\Delta f= \pm \frac{U_{c}}{l}
$$

Candel et al estimate that $U_{c} \approx 0.5 U_{J}$ and $l \approx 3.25 \delta$. Substituting these values into (110) leads to reasonably good estimates of the measured values of $\Delta f$ for each test case. In our prediction model, $\Delta f$ is given by (109), so we take $l \approx \delta$, which is smaller than that suggested by Candel, but leads to predictions of the 'double-hump' spectrum which have values of $\Delta f$ that are comparable with the measurements.

\section{CONCLUSIONS}

The spectral broadening effect of a turbulent shear layer has been modelled analytically with a high-frequency weak-scattering approach. The principal result of this work is equation (93), which gives an approximate expression of the far-field power spectrum of the scattered acoustic field. Comparison between analytic predictions, made with a frozen turbulence assumption, and experimental results, from an open flow wind tunnel, show that general trends in the data can be well predicted.

A key application of this result concerns radiation of turbine tones from the exhaust nozzle of a turbofan aeroengine: these tones propagate to the far field through one or more turbulent shear layers. Equation (93) provides a direct relationship between the field incident on the shear layer, due to the turbine tone, and the resulting scattered field. This result is however only valid for observers outside the cone of silence: the extension of the model to inside the cone of silence shall be presented in a future planned article.

It is important to note that the result derived here is valid only in the case of 'weak' scattering. In the model this is manifested through the fact that the source terms in the scattering equation are modelled using only the incident field, so interactions between the scattered field and the turbulence are ignored: this is a 'single-scattering' model. Physically, such a model will be valid in the case where, after haystacking has occured, the tone is still visible in the narrowband spectrum and is significantly higher that the broadband haystack level. However, it has been observed on certain engines at certain operating conditions that the haystacking may be so severe that the tone is no longer visible in the far-field spectrum, as all the tone energy has been scattered into the haystack. We refer to this as 'strong' scattering, which is beyond the limits of the current model to predict. Cargill [2] has proposed a model for strong scattering in a plane shear layer, which we intend to study and develop in the future.

\section{ACKNOWLEDGEMENTS}

The authors would like to acknowledge the contributions of the late Alex M. Cargill, who originally formulated the weak-scattering model used in this work while he was employed at Rolls-Royce plc.

The work was funded by the European sixth framework project TURNEX, coordinated by Dr B.J. Tester. 


\section{REFERENCES}

[1] Candel, S.M, Guedel, A., and Julienne, A., Refraction and Scattering of Sound in an Open Wind Tunnel Flow. Proceedings of the Sixth International Congress on Instrumentation in Aerospace Simulation Facilities, 1975, pp 288-300.

[2] Cargill, A.M., Theory for Sound Scattering for Turbulent Shear Layers. II: Scattering Theory. Rolls-Royce Theoretical Science Group Report TSG0457, 1989.

[3] Mathews, D.C., and Perachio, A.A., Progress in Core Engine and Turbine Noise Technology, AIAA paper 74-948, 1974.

[4] Mathews, D.C., Nagel, R.T., and Kester, J.D., Review of Theory and Methods for Turbine Noise Prediction, AIAA paper 75-540, 1975.

[5] Candel, S.M, Guedel, A., and Julienne, A., Radiation, Refraction and Scattering of Acoustic Waves in a Free Shear Flow. AIAA Paper AIAA-76-544, 1976.

[6] Candel, S.M., Julliand, M., and Julienne, A., Shielding and Scattering by a Jet Flow, AIAA Paper AIAA-76-545, 1976.

[7] Tatarski, V.I., Wave Propagation in a Turbulent Medium, Dover Publications, New York, 1961.

[8] Chernov, L.A., Wave Propagation in a Random Medium, Dover Publications, New York, 1960.

[9] Cargill, A.M., Sound Propagation Through Fluctuating Flows, AIAA Paper AIAA83-0697, 1983.

[10] Hardin, J.C., and Preisser, J.S., Stochastic Analysis of Spectral Broadening by a Free Turbulent Shear Layer, NASA Technical Paper 1816 (1981).

[11] Schmidt, D.W., and Tillman, P.M., Experimental Study of Sound-Wave Phase Fluctuations Caused by Turbulent Wakes, Journal of the Acoustical Society of America, 1970, 47, pp 310-1324.

[12] Campos, L.M.B.C., The Spectral Broadening of Sound by Turbulent Shear Layers. Part 1. The Transmission of Sound Through Turbulent Shear Layers. Journal of Fluid Mechanics, 1978, 89(4), pp 723-749.

[13] Campos, L.M.B.C., The Spectral Broadening of Sound by Turbulent Shear Layers. Part 2. The Spectral Broadening of Sound and Aircraft Noise. Journal of Fluid Mechanics, 1978, 89(4), pp 751-783.

[14] Brown, E.H., and Clifford, S.F., Spectral Broadening of an Acoustic Pulse Propagating Through Turbulence, Journal of the Acoustical Society of America, 1973, 54, pp 36-39.

[15] Ewert, R., Kornow, O., Tester, B.J., Powles, C.J., Delfs, J.W., and Rose, M., Spectral Broadening of Jet Engine Turbine Tones, AIAA Paper AIAA-2008-2940, 2008.

[16] McAlpine, A., Powles, C.J., and Tester, B.J., A Weak-Scattering Model for Tone Haystacking, AIAA Paper AIAA-2009-3216, 2009.

[17] Goldstein, M.E., Aeroacoustics, McGraw Hill, New York, 1976. 
[18] Wundrow, D.W., and Khavaran, A., On the Applicability of High-Frequency Approximations to Lilley's Equation, Journal of Sound and Vibration, 2004, 272, pp 793-830.

[19] Tester, B.J., and Morfey, C.L., Developments in Jet Noise Modeling - Theoretical Predictions and Comparisons with Measured Data, Journal of Sound and Vibration, 1976, 46, pp. 79-103.

[20] Bleistein, N., and Handelsman, R.A., Asymptotic Expansions of Integrals, Holt, Rinehart and Winston, New York, 1975.

[21] Batchelor, G.K., The Theory of Homogeneous Turbulence, Cambridge University Press, Cambridge, 1953.

[22] Ewert, R., Broadband Slat Noise Prediction Based on CAA and Stochastic Sound Sources From Fast Random-Particle Mesh (RPM) Method, Computers and Fluids, 2008, 37, pp 369-387. 